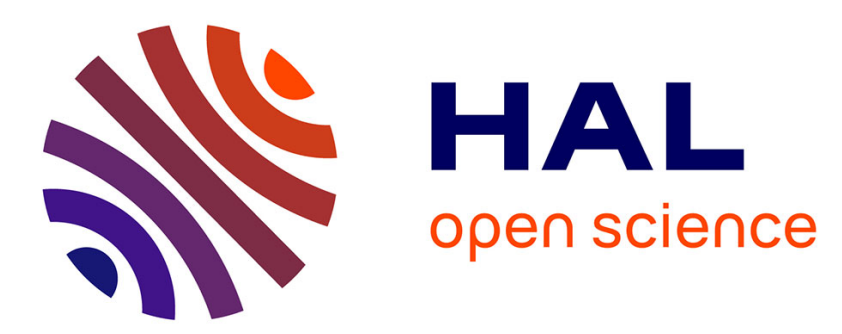

\title{
Bond graph formulation of an optimal control problem for linear time invariant systems
}

Wilfrid Marquis-Favre, Omar Mouhib, Bogdan Chereji, Daniel Thomasset, Jérôme Pousin, Martine Picq

\section{To cite this version:}

Wilfrid Marquis-Favre, Omar Mouhib, Bogdan Chereji, Daniel Thomasset, Jérôme Pousin, et al.. Bond graph formulation of an optimal control problem for linear time invariant systems. Journal of The Franklin Institute, 2008, 345 (4), pp.349-373. 10.1016/j.jfranklin.2007.10.005 . hal-00312905

\section{HAL Id: hal-00312905 https://hal.science/hal-00312905}

Submitted on 29 Mar 2019

HAL is a multi-disciplinary open access archive for the deposit and dissemination of scientific research documents, whether they are published or not. The documents may come from teaching and research institutions in France or abroad, or from public or private research centers.
L'archive ouverte pluridisciplinaire $\mathbf{H A L}$, est destinée au dépôt et à la diffusion de documents scientifiques de niveau recherche, publiés ou non, émanant des établissements d'enseignement et de recherche français ou étrangers, des laboratoires publics ou privés. 


\title{
Bond graph formulation of an optimal control problem for linear time-invariant systems
}

\author{
†Wilfrid Marquis-Favre, Omar Mouhib, Bogdan Chereji, Daniel Thomasset \\ *Jérôme Pousin, Martine Picq \\ ${ }^{\dagger}$ Laboratoire AMPERE \\ *Institut Camille Jordan \\ Bât. St Exupéry \\ 25, avenue Jean Capelle \\ Bât. Léonard de Vinci \\ Institut National des sciences Appliquées de Lyon \\ F-69621 Villeurbanne Cedex \\ e-mails: < firstname $>$.<lastname $>@$ insa-lyon.fr
}

\begin{abstract}
A recent communication has proposed a conjectural procedure for representing a category of optimal control problems in bond graph language [1]. This paper aims at providing a fundamental theory for proving the effectiveness of this procedure. The class of problem that the procedure can deal with has been extended. Its application was formerly restricted to linear time invariant siso system. The systems considered now are linear time invariant MIMO systems. The optimisation objective is the minimization of dissipation and input. The developments concerning the optimal control problem are based on the Pontryagin Maximum Principle and the proof of the effectiveness of the procedure makes a broad use of the port-Hamiltonian concept. As a result, the bond graph representation of the given optimisation problem enables the analytical system, which provides the optimal solution, to be derived. The work presented in this paper is the first step in research with perspectives towards formulating dynamic optimisation problems in bond graph and, towards coupling this formulation with a sizing methodology using bond graph language and a state-space inverse model approach. This sizing methodology, however, is not the topic of this paper and thus is not presented here.
\end{abstract}

Keywords: Optimal control, bond graph, Pontryagin Maximum Principle, port-Hamiltonian system, bicausality.

\section{Nomenclature}

‘T,

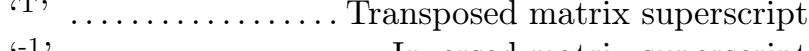

$\ldots \ldots \ldots$ Inversed matrix superscript ' $\mathrm{r}, \mathrm{c}$ '........ Resistance, conductance port indices $\dot{a} \ldots \ldots \ldots \ldots$ Time derivative of $a$ $\frac{\partial A}{\partial \boldsymbol{x}} \ldots \ldots$ Gradient operator on $A$ with respect to $\boldsymbol{x}$ $\frac{\partial^{2} A}{\partial \boldsymbol{a} \partial \boldsymbol{b}} \ldots$. Second partial derivative of $A$ with respect to $\boldsymbol{a}$ and $\boldsymbol{b}$ components $\frac{\partial \boldsymbol{a}}{\partial \boldsymbol{x}}$... Matrix composed in rows by the gradients of the $\boldsymbol{a}$ components with respect to $\boldsymbol{x}$ $\operatorname{tr} \ldots \ldots \ldots \ldots \ldots \ldots \ldots . \ldots . \ldots . \ldots . \ldots$ Matrix trace $t_{0}, t_{f} \ldots \ldots \ldots \ldots \ldots \ldots \ldots$ Initial and final times $\boldsymbol{x} \ldots \ldots \ldots \ldots \ldots \ldots \ldots \ldots \ldots \ldots \ldots \ldots$ State vector $\boldsymbol{x}_{0}, \boldsymbol{x}_{f} \ldots \ldots \ldots \ldots$. Initial and final state vectors
$\boldsymbol{x}_{\mathrm{I}}, \boldsymbol{x}_{\mathrm{C}} \ldots \ldots$ I-element, C-element energy variable $\lambda$.......... Lagrange multiplier or co-state vector $\boldsymbol{x}_{\lambda} \ldots \ldots \ldots \ldots \ldots \ldots$ Optimizing-state vector $\boldsymbol{x}_{\lambda \mathrm{I}}, \boldsymbol{x}_{\lambda \mathrm{C}} \ldots$ I-element, C-element optimizing-state vectors

$\boldsymbol{\Lambda}_{x}, \boldsymbol{\Lambda}_{\lambda} \ldots$ Terms of the optimizing-state equations $\lambda_{\mathrm{I}}, \boldsymbol{\lambda}_{\mathrm{C}} \ldots \ldots$ I-element, C-element co-state vectors $\boldsymbol{u}, \boldsymbol{y} \ldots \ldots \ldots \ldots \ldots \ldots$ Input, output vectors $\boldsymbol{u}_{\mathrm{R}}, \boldsymbol{y}_{\mathrm{R}} \ldots$ Input, output vectors associated to the dissipative phenomena $\boldsymbol{u}_{e}, \boldsymbol{u}_{f} \ldots \ldots \ldots \ldots \ldots$ Effort, flow input vectors $\boldsymbol{u}_{\mathrm{opt}} \ldots \ldots \ldots \ldots$ Optimal input solution vector $\boldsymbol{e}, \boldsymbol{f} \ldots \ldots \ldots \ldots \ldots \ldots \ldots \ldots$ Effort, flow vectors 
$\boldsymbol{e}_{\mathrm{R}}, \boldsymbol{f}_{\mathrm{R}} \ldots \ldots$ Effort, flow vectors of R-elements in the dualized representations $\boldsymbol{e}_{\mathrm{opt}}, \boldsymbol{f}_{\mathrm{opt}} \ldots$ Effort, flow vectors in the optimizing bond graph $V \ldots \ldots \ldots \ldots \ldots \ldots$. . . . . . . . . $\mathcal{L} \ldots \ldots \ldots \ldots \ldots \ldots \ldots \ldots \ldots \ldots$ Lagrangian $H \ldots \ldots \ldots \ldots \ldots \ldots \ldots \ldots \ldots$ Hamiltonian $P_{\text {diss }} \ldots \ldots \ldots \ldots \ldots \ldots$ Dissipation power $H_{\mathrm{p}} \ldots \ldots \ldots \ldots \ldots \ldots \ldots \ldots$ Pontryagin function I $\ldots \ldots \ldots \ldots \ldots \ldots \ldots \ldots \ldots$ Identity matrix $\mathbf{H} \ldots \ldots \ldots \ldots \ldots \ldots \ldots$ Hessian matrix of $H$ $\mathbf{R} \ldots \ldots \ldots \ldots$ R-element characteristic matrix $\mathbf{R}_{u} \ldots \ldots \ldots$ Control weighted matrix on inputs $\mathbf{R}_{u_{e}}, \mathbf{R}_{u_{f}} \ldots \ldots$. Control weighted matrices on effort, flow inputs
$\mathbf{R}_{\mathbf{R}} \ldots$.... R-element characteristic matrix in dualized representations T ...... Coupling R-element characteristic matrix J ...... Poisson Structure matrix associated to the junction structure S ... Matrix associated with the R-elements and the junction structure transformations g .....Matrix associated with the junction structure transformations between the storage elements and the sources

$\mathbf{g}_{\mathbf{R}}$... Matrix associated with the junction structure transformations between the storage elements and the R-elements in dualized representations П ...... Matrix of the second derivatives of $H_{\mathrm{p}}$ with respect to the $\boldsymbol{x}$ and $\boldsymbol{u}$ components

\section{Introduction}

Dynamic optimisation involves systems where the variables are functions of time and where the models are governed by Differential- (either Ordinary or Algebraic) Equations [2, 3, 4]. Also, in many optimisation problems, the performance index ${ }^{1}$ is energy, state and/or input based. There is a justification for raising the question: "to what extent bond graph language, that straightforwardly displays both the dynamics and the energy topology of a system model, can be used for representing the formulation of an optimisation problem?". In a bond graph language context, research dealing with optimisation have principally a sensitivity approach $[5,6,7,8,9]$.

Introducing optimisation into bond graph language brings more insight and a new vision about optimisation as can be experienced through this paper. Moreover coupling optimisation and bond graph has also System Engineering arguments. In fact a perspective is to extend a methodology concerning mechatronic system sizing on dynamic and energy criteria $[10,11,12,13]$. With this in view, integration of the actuating line component specifications, optimal control, multivariable control, energy minimization in a context of sustainable development, design specifications not precisely defined, and structure synthesis, all the aforementioned are expected to be dealt with. Optimal control is the first step in this perspective of introducing a more general optimisation problem into bond graph language and, concerning this, a conjectural procedure has been recently proposed [1]. The objective of the present paper is to provide a theoretical framework for proving the effectiveness of this procedure.

The proposed procedure concerns the optimal control of linear time invariant systems where the performance index is an integral of a certain functional. Several approaches such as variational methods, dynamic programing or gradient methods exist to deal with this category of optimisation problem [3]. The developments presented here are based on the variational methods and more precisely on the Pontryagin Maximum Principle. They aim at providing a bond graph representation that provides the analytical system of equations giving the optimal control solution. So the main contribution of the present work is to provide a new way for formulating this analytical system and this new way is based on bond graph language. As already mentioned the optimisation problem undertaken corresponds to finding the optimal control of a linear time invariant MIMO system. The integral performance index is based on inputs and dissipation energy. Boundary conditions are supposed fixed, in particular for both final time and final state, and finally no constraint exists on the inputs or the state. This voluntary restricted hypothesis framework has enabled the first step in the coupling of optimisation and bond graph to be clearly investigated and offers encouraging perspectives for future works.

The next section (Section 2) gives the procedure for building the bond graph representation corresponding to the given optimal control problem. Section 3 gives a proposition and its demonstration that justify the

\footnotetext{
${ }^{1}$ Although historically speaking the terminology is closely related to the application domain, the terms 'performance index', 'cost-function' and 'objective-function' can be indifferently used for designating the criterion that is to be optimized [3].
} 
former procedure and prove its effectiveness. The key idea of the proof is to apply the Pontryagin Maximum Principle to a generic port-Hamiltonian system. The port-Hamiltonian system is an analytical expression of the dynamic equations governing a model, and clearly and mathematically reflects the energy topology of the system model. Then the proposed procedure is illustrated in section 4 using a DC motor example. Section 5 draws three important features of the proposed work from the optimisation point of view. These features concern the extremum sufficient condition, the general unstability property of the formulated optimal system and the form of the differential equation system obtained. Finally the conclusion (section 6) summarizes the present work and gives some elements for future developments.

Additionally, two appendices give, respectively, bases on partial dualization in the bond graph used for the procedure effectiveness proof (appendix A) and on the bicausality concept for the bond graph exploitation that gives the optimal control system (appendix B).

\section{Procedure for the bond graph formulation of an optimal control problem}

Starting from the bond graph of a model, the object of the optimal control problem, the procedure presented here enables an augmented bond graph to be set up. This augmented bond graph consists of the original model representation coupled to an optimizing bond graph. It furnishes, by its bicausal exploitation, the set of differential-algebraic equations that gives the solution to the optimal control problem. The procedure is stated as follows:

Given:

- a linear time invariant model of a MIMO system and its bond graph representation,

- the input controls to determine with respect to the integral performance index (1) to minimize, that is the integral of half a quadratic form based on dissipative energy and control,

- fixed-boundary conditions for the time and state space, and

- no constraint on inputs or state,

the following procedure enables the bond graph representation of the given optimal control problem to be obtained.

\section{Procedure: Optimal control bond graph construction}

1. For the input controls to be determined, add to the model bond graph a multiport R-element characterized by the control weighted matrix in the performance index. This multiport R-element is connected to a junction array inserted into the control source bond and corresponding to the variable nature of the control i.e. a 0 (resp. 1 or combined 0 and 1)-junction array for effort (resp. flow or combined effort and flow) variables.

2. Duplicate the model bond graph with its element parameters except for the multiport R-elements. For the multiport R-elements, the characteristic matrices are transposed and reversed sign. The duplicated representation is hereafter called the optimizing bond graph.

3. For the dissipative phenomena involved in the integral performance index, couple the corresponding multiport R-elements respectively in the model and the optimizing bond graphs. Add the matrix $\frac{1}{2}[\mathbf{R}+\mathbf{T}]$ as the lower extra diagonal submatrix. $\mathbf{R}=\left[\begin{array}{ll}\mathbf{R}_{\mathrm{rr}} & \mathbf{R}_{\mathrm{rc}} \\ \mathbf{R}_{\mathrm{cr}} & \mathbf{R}_{\mathrm{cc}}\end{array}\right]$ (r-resp. c- corresponding to the ports in resistance - resp. conductance- causality - see appendix A- when the bond graph is in integral causality), this $\mathbf{R}$ is the characteristic matrix of the corresponding multiport R-elements in the model bond graph and $\mathbf{T}=\left[\begin{array}{cc}\mathbf{R}_{\mathrm{rr}}^{\mathrm{T}} & -\mathbf{R}_{\mathrm{cr}}^{\mathrm{T}} \\ -\mathbf{R}_{\mathrm{rc}}^{\mathrm{T}} & \mathbf{R}_{\mathrm{cc}}^{\mathrm{T}}\end{array}\right]$. In the case of the 1-port R-elements, the lower extra diagonal matrix is simply constituted by the model R-element parameters in its diagonal.

4. For the input controls to be determined, couple the multiport R-elements added at step 1 respectively in the model and the optimizing bond graphs by setting the lower extra diagonal submatrix to the control weighted matrix. 
5. Replace, in the model bond graph, the source elements involved in the input controls to be determined by double detectors and, in the optimizing bond graph, by double sources. The double sources impose both null efforts and flows.

6. Assign bicausality to the bond graph obtained. Bicausality propagates from the double sources to the double detectors through the R-elements added at step 1. The analytical exploitation of the bicausal bond graph representation obtained provides the equation system and the optimal control solution to the given problem.

\section{End of the procedure}

In this procedure, concerning the coupling R-elements, the following convention has been adopted. The first half of the components of the conjugate variable vectors correspond to the model bond graph while the second half corresponds to the optimizing bond graph.

Before presenting a proof of the effectiveness of the procedure, a very simple example is now presented to briefly illustrate the different steps of the bond graph construction (steps 1-5). The bond graph representation of the chosen example is shown in Fig. 1-a. It represents a simple second order model like, for instance, a RLC-circuit fed by a voltage source in the electrical domain or a spring-damper-mass with a force applied to the mass in the mechanical domain. The graphical steps are illustrated respectively in Fig. 1-b to f. The Fig. 1-b shows the introduction of an R-element onto a 0-junction between the effort source and the rest of the model bond graph. This may be interpreted as a dissipation of the energy supply device or as taking into account a control weighted factor. The added R-element does not change the dynamic behaviour of the original model at all. Then the model bond graph is duplicated into the optimizing bond graph (Fig. 1-c) where the R-elements are characterized by a reversed sign parameter. The right-hand side R-element is replaced by a global 2-port R-element where the lower extra diagonal coefficient is set to R (Fig. 1-d). In Fig. 1-e the same operation is applied to the left-hand side R-element. Finally the step before the final one shown in Fig. 1-f shows the replacement of the effort source by a double source in the optimizing bond graph and by a double detector in the model bond graph.

Since the objective of this small first example is only to illustrate the graphical operations of the procedure, no bicausal exploitation has been undertaken at this stage. The corresponding step 6 is applied to the section 4 example which illustrates this. For a brief introduction to bicausality, see appendix B.

\section{Proof of the effectiveness of the procedure}

The objective of this section is to prove that the bond graph representation obtained by the above procedure corresponds well to the given optimal control problem. Firstly a proposition concerning this results is given. The proof of this proposition is based on the port-Hamiltonian system concept $[14,15,16,17]$. This concept is well adapted to the demonstration of the procedure effectiveness since the port-Hamiltonian system can be viewed as the geometric counterpart of a graphical bond graph representation. This mathematical formulation is mid-way between a global formulation, such as the one of the state space equations, and the energy-based graphical representation of bond graph language. Thus a presentation of the port-Hamiltonian formulation is given in the following proof framework. Then the Pontryagin Maximum Principle is recalled as the optimum control formulation. Finally the proof of the proposition is developed by applying the Pontryagin method to the port-Hamiltonian formulation.

\section{Proposition}

Given a linear time invariant model with its bond graph representation (Fig. 2-a) and subject to an optimal control problem with input and dissipation-based integral performance index of the form (1); and given the boundary conditions of this optimal control problem; the bond graph representation, whose the analytical exploitation provides the set of differential-algebraic equations that gives the solution of the given optimal problem, is displayed in Fig. 2-b where the junction structure and the multiport IC-element are identical.

$$
V=\int_{\mathrm{t}_{0}}^{\mathrm{t}_{\mathrm{f}}} \frac{1}{2}\left(\boldsymbol{u}_{e}^{\mathrm{T}} \cdot \mathbf{R}_{u_{e}}^{-1} \cdot \boldsymbol{u}_{e}+\boldsymbol{u}_{f}^{\mathrm{T}} \cdot \mathbf{R}_{u_{f}} \cdot \boldsymbol{u}_{f}+P_{\mathrm{diss}}\right) d t
$$



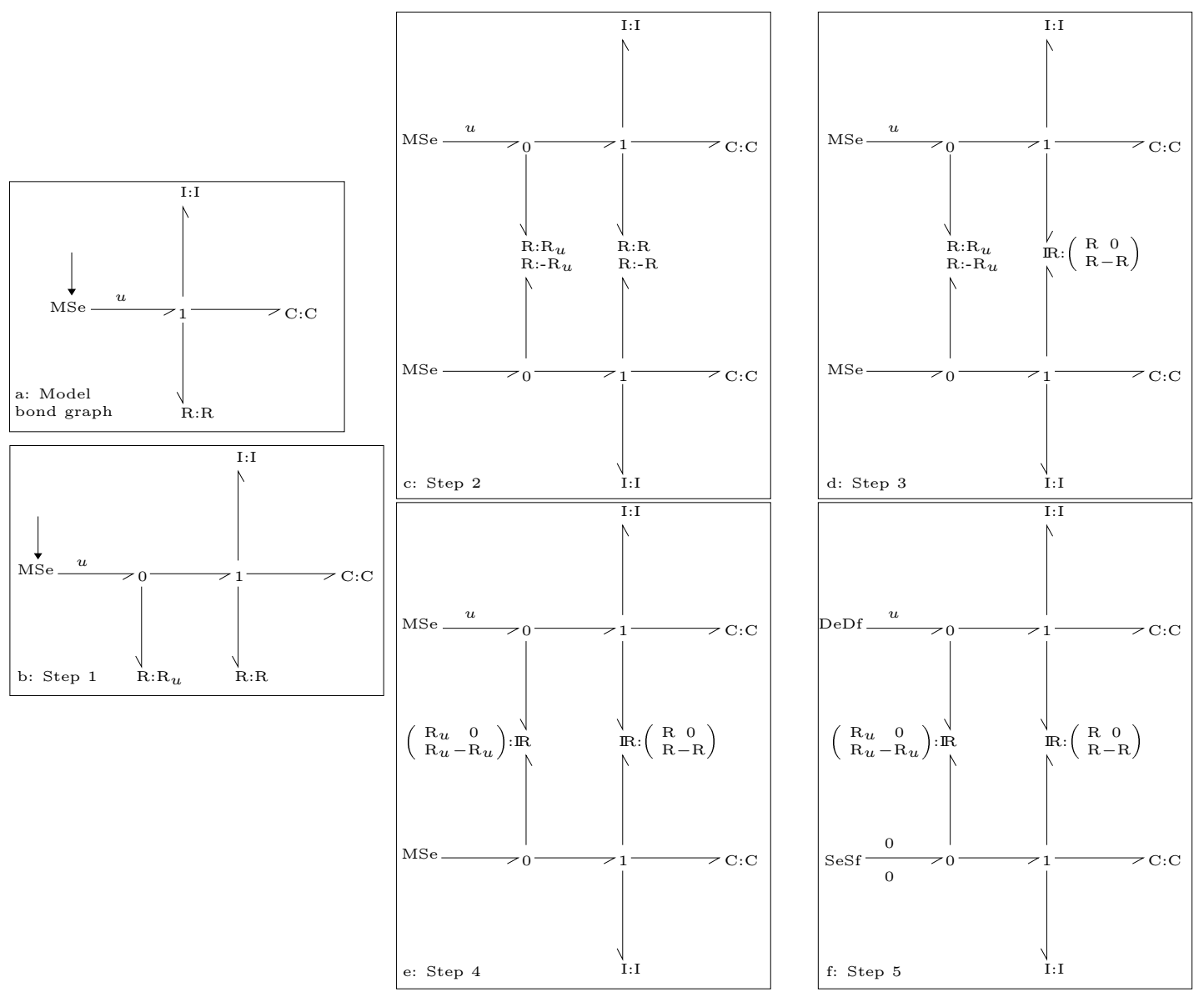

Figure 1: Illustration of the procedure 1 to 5 steps

where $\mathbf{R}_{u_{e}}$ and $\mathbf{R}_{u_{f}}$ are control weighted matrices, $P_{\text {diss }}$ is the dissipation power at certain multiport R-element ports, $\mathbf{T}=\left[\begin{array}{cc}\mathbf{R}_{\mathrm{rr}}^{\mathrm{T}} & -\mathbf{R}_{\mathrm{cr}}^{\mathrm{T}} \\ -\mathbf{R}_{\mathrm{rc}}^{\mathrm{T}} & \mathbf{R}_{\mathrm{cc}}^{\mathrm{T}}\end{array}\right]$ (with $\mathbf{R}=\left[\begin{array}{ll}\mathbf{R}_{\mathrm{rr}} & \mathbf{R}_{\mathrm{rc}} \\ \mathbf{R}_{\mathrm{cr}} & \mathbf{R}_{\mathrm{cc}}\end{array}\right]$, r -resp. c- corresponding to R-element ports in resistance -resp. conductance- causality -see apendix A- when the bond graph is in integral causality). A multibond graph notation has been adopted [18] where GJS stands for Generalized Junction Structure.

\section{Proof of the proposition}

\section{Port-Hamiltonian system}

Consider a system with the total stored energy represented by its Hamiltonian $H$. $H$ is expressed in the linear case as a quadratic form of $\boldsymbol{x} \in \mathbb{R}^{\mathrm{n}}$, the state space vector corresponding to the energy variables in bond graph language (equation 2). $H$ represents the total stored energy in the system and by taking appropriate references, it is supposed that $H(\boldsymbol{x})$ is strictly positive. Furthermore the Hessian matrix $\mathbf{H}$ of the Hamiltonian $H$ is symmetric $\left(\mathbf{H}=\mathbf{H}^{\mathrm{T}}\right)$ due to the reversibility property and also because it verifies the Maxwell reciprocity property [19, 20]. With the previous hypothesis about $H, \mathbf{H}$ is also definite positive.

$$
\begin{aligned}
H: \mathbb{R}^{\mathrm{n}} & \longrightarrow \mathbb{R} \\
\boldsymbol{x} & \longmapsto H(\boldsymbol{x})=\frac{1}{2} \boldsymbol{x}^{\mathrm{T}} \cdot \mathbf{H} \cdot \boldsymbol{x}
\end{aligned}
$$

where the superscript ' $\mathrm{T}$ ' denotes the symbol of a transposed matrix or vector. 

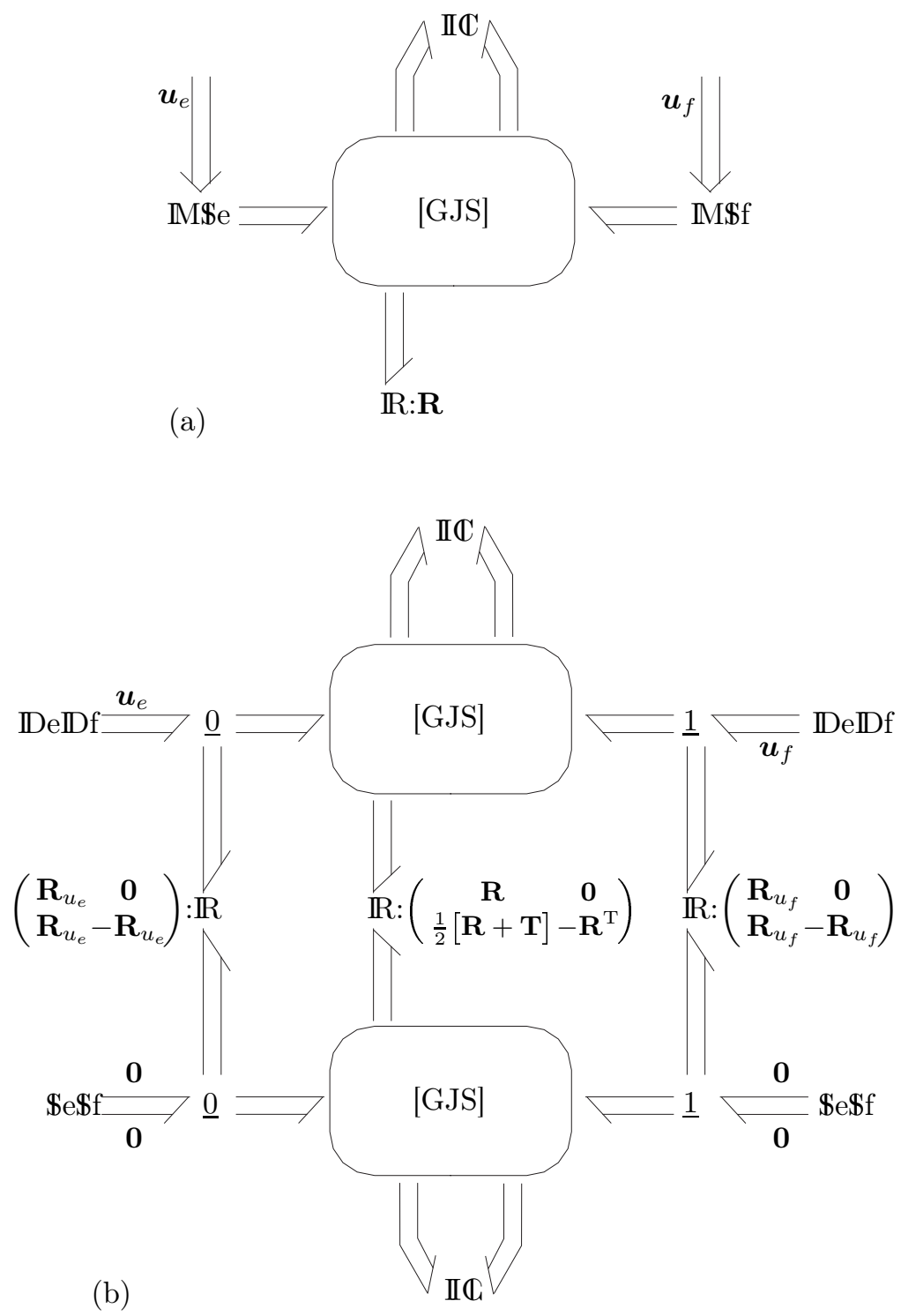

Figure 2: (a) model bond graph and (b) optimal control bond graph 
First port-Hamiltonian systems are introduced for conservative, i.e. not dissipative, models. In the hypothesis framework of linear time invariant systems, they are given by $(3)[14,16,17]$.

$$
\left\{\begin{array}{l}
\dot{\boldsymbol{x}}=\mathbf{J} \cdot \frac{\partial H(\boldsymbol{x})}{\partial \boldsymbol{x}}+\mathbf{g} \cdot \boldsymbol{u} \\
\boldsymbol{y}=\mathbf{g}^{\mathrm{T}} \cdot \frac{\partial H(\boldsymbol{x})}{\partial \boldsymbol{x}}
\end{array}\right.
$$

where $\mathbf{J}$ and $\mathbf{g}$ are constant matrices associated to junction structure transformations in the bond graph, $\boldsymbol{u}=\left[\begin{array}{ll}\boldsymbol{u}_{e}^{\mathrm{T}} & \boldsymbol{u}_{f}^{\mathrm{T}}\end{array}\right]^{\mathrm{T}} \in \mathbb{R}^{\mathrm{m}}$ and $\boldsymbol{y} \in \mathbb{R}^{\mathrm{m}}$ are respectively the system input and output vectors, and $\frac{\partial \text {. }}{\partial \boldsymbol{x}}$ denotes the gradient operator with respect to the vector $\boldsymbol{x}$.

In the port-Hamiltonian system framework, $\boldsymbol{u}$ and $\boldsymbol{y}$ are the power conjugate variable vectors at the environmental ports of the system. Also $\mathbf{J}$ is a Poisson Structure matrix satisfying the skew-symmetry property i.e. $\mathbf{J}=-\mathbf{J}^{\mathrm{T}}[15]$. One peculiarity of the port-Hamiltonian description is to clearly display the energy topology of the physical model in a mathematical manner. If dissipative phenomena are considered, the port-Hamiltonian system is now supposed to be written:

$$
\left\{\begin{array}{l}
\dot{\boldsymbol{x}}=\mathbf{J} \cdot \frac{\partial H(\boldsymbol{x})}{\partial \boldsymbol{x}}+\mathbf{g} \cdot \boldsymbol{u}-\mathbf{g}_{\mathrm{R}} \cdot \boldsymbol{u}_{\mathrm{R}} \\
\boldsymbol{y}=\mathbf{g}^{\mathrm{T}} \cdot \frac{\partial H(\boldsymbol{x})}{\partial \boldsymbol{x}} \\
\boldsymbol{y}_{\mathrm{R}}=\mathbf{g}_{\mathrm{R}}^{\mathrm{T}} \cdot \frac{\partial H(\boldsymbol{x})}{\partial \boldsymbol{x}}
\end{array}\right.
$$

where $\boldsymbol{u}_{\mathrm{R}} \in \mathbb{R}^{\mathrm{r}}$ and $\boldsymbol{y}_{\mathrm{R}} \in \mathbb{R}^{\mathrm{r}}$ are the input and output vectors associated to the dissipative phenomena, and $\mathbf{g}_{\mathrm{R}}$ is a matrix associated to the junction structure transformation between the storage elements and the R-elements.

By an appropriate dualization of the dissipative constitutive laws, it is always possible to consider that the inputs are efforts $\left(\boldsymbol{u}_{\mathrm{R}}=\boldsymbol{e}_{\mathrm{R}}\right)$ while the outputs are flows $\left(\boldsymbol{y}_{\mathrm{R}}=\boldsymbol{f}_{\mathrm{R}}\right)$. Thus, in the linear case, the dissipative constitutive laws can be written:

$$
e_{\mathrm{R}}=\mathbf{R}_{\mathrm{R}} \cdot \boldsymbol{f}_{\mathrm{R}}
$$

where $\mathbf{R}_{\mathrm{R}}$ is the matrix that characterizes the dissipative phenomena.

A generic causal bond graph representation of equations (4) and (5) is given in Fig. 3 where $\boldsymbol{x}=\left[\boldsymbol{x}_{\mathrm{I}}^{\mathrm{T}} \boldsymbol{x}_{\mathrm{C}}^{\mathrm{T}}\right]^{\mathrm{T}}$. For the sake of conciseness, partial dualization has been used for R-element ports with conductance causality and the multiport flow source [21]. This introduces symplectic gyrators that inverse the effort and flow roles and enable every case of port element causality assignment, for multiport R- and source elements, in a unique causal representation to be treated, without loss of generality, in the demonstration (see appendix A). In figure 3 the symplectic gyrators are encapsulated in the word [GJS]. From equation (2), it can be deduced that:

$$
\frac{\partial H(\boldsymbol{x})}{\partial \boldsymbol{x}}=\mathbf{H} \cdot \boldsymbol{x}
$$

Also, the vector $\boldsymbol{f}_{\mathrm{R}}$ can now be expressed in terms of the state vector:

$$
\boldsymbol{f}_{\mathrm{R}}=\mathbf{g}_{\mathrm{R}}^{\mathrm{T}} \cdot \frac{\partial H(\boldsymbol{x})}{\partial \boldsymbol{x}}=\mathbf{g}_{\mathrm{R}}^{\mathrm{T}} \cdot \mathbf{H} \cdot \boldsymbol{x}
$$

Due to dualization the matrix $\mathbf{R}_{\mathrm{R}}$, that characterizes the multiport R-element in the figure 3 generic causal bond graph, differs from the matrix of the figure 2-a multiport R-element in the original bond graph.

Finally the port-Hamiltonian system for a dissipative linear time invariant model can be written:

$$
\left\{\begin{array}{l}
\dot{\boldsymbol{x}}=[\mathbf{J}-\mathbf{S}] \cdot \mathbf{H} \cdot \boldsymbol{x}+\mathbf{g} \cdot \boldsymbol{u} \\
\boldsymbol{y}=\mathbf{g}^{\mathrm{T}} \cdot \mathbf{H} \cdot \boldsymbol{x}
\end{array}\right.
$$

where $\mathbf{S}$, given by equation (9), is a positive definite matrix if $\mathbf{R}_{\mathrm{R}}$ is also a positive definite matrix.

$$
\mathbf{S}=\mathbf{g}_{\mathrm{R}} \cdot \mathbf{R}_{\mathrm{R}} \cdot \mathbf{g}_{\mathrm{R}}^{\mathrm{T}}
$$

Now the method of the Pontryagin Maximum principle will be recalled. 


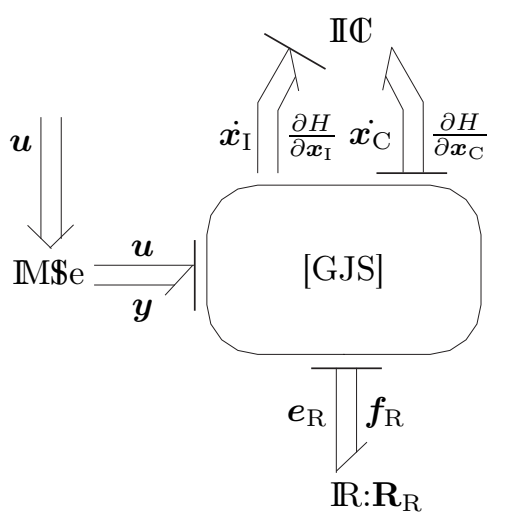

Figure 3: Generic causal bond graph representation for a port-Hamiltonian system

\section{Pontryagin Maximum Principle}

Consider the state space model (10) and the integral performance index or cost function $V$ to minimize (11) $[22,23,4]$.

$$
\begin{aligned}
& \dot{\boldsymbol{x}}=\boldsymbol{f}(\boldsymbol{x}, \boldsymbol{u}) \\
& V=\int_{\mathrm{t}_{0}}^{\mathrm{t}_{\mathrm{f}}} \mathcal{L}(\boldsymbol{x}, \boldsymbol{u}) d t
\end{aligned}
$$

where $\boldsymbol{x} \in \mathbb{R}^{\mathrm{n}}$ is the state space vector, $\boldsymbol{u} \in \mathbb{R}^{\mathrm{m}}$ is the control vector where each component here is supposed unbounded, $\boldsymbol{f}$ is a differentiable function of $\boldsymbol{x}$ and $\boldsymbol{u}$, and $\mathcal{L}$, the Lagrangian, is supposed to be a positive definite function of $\boldsymbol{x}$ and $\boldsymbol{u}$. The integral performance index minimization is considered between the given initial state $\mathbf{x}_{0}=\mathbf{x}\left(\mathrm{t}_{0}\right)$ at time $\mathrm{t}_{0}$ and final state $\mathbf{x}_{\mathrm{f}}=\mathbf{x}\left(\mathrm{t}_{\mathrm{f}}\right)$ at time $\mathrm{t}_{\mathrm{f}}$.

The first step of the Pontryagin method is to build the Pontryagin function $H_{\mathrm{p}}$ given by:

$$
\begin{aligned}
H_{\mathrm{p}}: \quad \mathbb{R}^{\mathrm{n}} \times \mathbb{R}^{\mathrm{n}} \times \mathbb{R}^{\mathrm{m}} & \longrightarrow \mathbb{R} \\
(\boldsymbol{x}, \boldsymbol{\lambda}, \boldsymbol{u}) & \longmapsto H_{\mathrm{p}}(\boldsymbol{x}, \boldsymbol{\lambda}, \boldsymbol{u})=\mathcal{L}(\boldsymbol{x}, \boldsymbol{u})+\boldsymbol{\lambda}^{\mathrm{T}} \cdot \boldsymbol{f}(\boldsymbol{x}, \boldsymbol{u})
\end{aligned}
$$

where $\boldsymbol{\lambda} \in \mathbb{R}^{\mathrm{n}}$ is the vector of co-state variables (or covariant vector) usually called Lagrange multiplier vector of the associated constrained variational problem, and the subscript 'p' distinguishes the Pontryagin function of the optimisation problem from the Hamiltonian of the system.

The set of $2 \mathrm{n}+\mathrm{m}$ first order differential-algebraic equations (13) provides the optimal solution for $\boldsymbol{x}$ (nstate equations), $\boldsymbol{\lambda}$ (n-costate equations) and $\boldsymbol{u}$ (m Euler equations in terms of the vector $\boldsymbol{u}$ components) with the boundary conditions $\mathbf{x}_{0}$ and $\mathbf{x}_{\mathrm{f}}$.

$$
\left\{\begin{array}{l}
\dot{\boldsymbol{x}}=\frac{\partial H_{\mathrm{p}}(\boldsymbol{x}, \boldsymbol{\lambda}, \boldsymbol{u})}{\partial \boldsymbol{\lambda}} \\
\dot{\boldsymbol{\lambda}}=-\frac{\partial H_{\mathrm{p}}(\boldsymbol{x}, \boldsymbol{\lambda}, \boldsymbol{u})}{\partial \boldsymbol{x}} \\
\frac{\partial H_{\mathrm{p}}(\boldsymbol{x}, \boldsymbol{\lambda}, \boldsymbol{u})}{\partial \boldsymbol{u}}=\mathbf{0}
\end{array}\right.
$$

Unlike the classical presentation of the Pontryagin Maximum Principle, it is chosen not to calculate $a$ priori the optimal controls from the Euler equations in terms of the vector $\boldsymbol{u}$ components. It is shown below why it is preferable here.

The last step of the demonstration is the application of the Pontryagin Maximum Principle to the equation (8) system. 


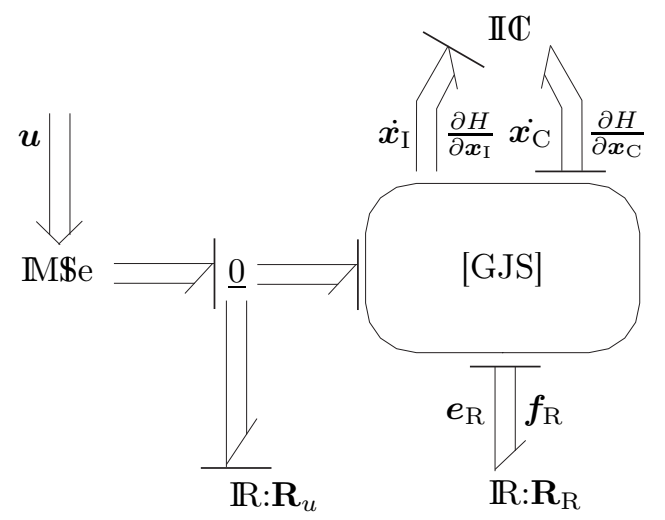

Figure 4: Generic causal bond graph representation for a port-Hamiltonian system with non-ideal energy supplies

\section{Application of the Pontryagin Maximum Principle to the port-Hamiltonian systems}

The integral performance index considered here corresponds to the minimization of certain energy dissipation and inputs. Equation (11) in this case becomes:

$$
V=\int_{\mathrm{t}_{0}}^{\mathrm{t}_{\mathrm{f}}} \frac{1}{2}\left(\boldsymbol{u}^{\mathrm{T}} \cdot \mathbf{R}_{u}^{-1} \cdot \boldsymbol{u}+P_{\mathrm{diss}}\right) d t=\int_{\mathrm{t}_{0}}^{\mathrm{t}_{\mathrm{f}}} \frac{1}{2}\left(\boldsymbol{u}^{\mathrm{T}} \cdot \mathbf{R}_{u}^{-1} \cdot \boldsymbol{u}+\boldsymbol{e}_{\mathrm{R}}^{\mathrm{T}} \cdot \boldsymbol{f}_{\mathrm{R}}\right) d t
$$

where $\mathbf{R}_{u}$ is a diagonal matrix of positive elements that represent control weighted factors, and $P_{\text {diss }}$ is the dissipation power expressed as the inner product of the power conjugate vectors of the R-elements. The coefficient $\frac{1}{2}$, without changing the optimisation problem, enables a coefficient 2 in certain terms in the following to be eliminated.

The matrix $\mathbf{R}_{u}$ may be interpreted as characterizing some dissipative phenomena embedded in the energy supplies thus considered as non-ideal [4]. The bond graph implementation is displayed in Fig. 4.

The Pontryagin function (12) applied to the port-Hamiltonian system (8) with the equation (5) and the integral performance index (14) gives:

$$
H_{\mathrm{p}}=\frac{1}{2} \boldsymbol{u}^{\mathrm{T}} \cdot \mathbf{R}_{u}^{-1} \cdot \boldsymbol{u}+\frac{1}{2} \boldsymbol{f}_{\mathrm{R}}^{\mathrm{T}} \cdot \mathbf{R}_{\mathrm{R}} \cdot \boldsymbol{f}_{\mathrm{R}}+\boldsymbol{\lambda}^{\mathrm{T}} \cdot[[\mathbf{J}-\mathbf{S}] \cdot \mathbf{H} \cdot \boldsymbol{x}+\mathbf{g} \cdot \boldsymbol{u}]
$$

From this expression the set of differential-algebraic equations (13) providing the optimal solution are now derived:

$$
\begin{aligned}
& \dot{\boldsymbol{x}}=[\mathbf{J}-\mathbf{S}] \cdot \mathbf{H} \cdot \boldsymbol{x}+\mathbf{g} \cdot \boldsymbol{u} \\
& \dot{\boldsymbol{\lambda}}=-\frac{\partial}{\partial \boldsymbol{x}}\left[\frac{1}{2} \boldsymbol{f}_{\mathrm{R}}^{\mathrm{T}} \cdot \mathbf{R}_{\mathrm{R}} \cdot \boldsymbol{f}_{\mathrm{R}}\right]-\left[\boldsymbol{\lambda}^{\mathrm{T}} \cdot[\mathbf{J}-\mathbf{S}] \cdot \mathbf{H}\right]^{\mathrm{T}} \\
& \mathbf{R}_{u}^{-1} \cdot \boldsymbol{u}+\left[\boldsymbol{\lambda}^{\mathrm{T}} \cdot \mathbf{g}\right]^{\mathrm{T}}=\mathbf{0}
\end{aligned}
$$

While equation (16) can be derived from the Fig. 4 bond graph representation, the key issue of the bond graph formulation of the optimal control problem under consideration resides in the translation of equations (17) and (18) into this language.

Let us first concentrate on equation (17). It can be re-written using the symmetry and skew-symmetry properties of matrices $\mathbf{H}$ and $\mathbf{J}$ respectively, also using equation (7), and the expression (9) of matrix $\mathbf{S}$ as the equation (19) shows.

$$
\begin{aligned}
\dot{\boldsymbol{\lambda}} & =-\frac{\partial \boldsymbol{f}_{\mathrm{R}}^{\mathrm{T}}}{\partial \boldsymbol{x}} \cdot \frac{1}{2}\left[\mathbf{R}_{\mathrm{R}}+\mathbf{R}_{\mathrm{R}}^{\mathrm{T}}\right] \cdot \boldsymbol{f}_{\mathrm{R}}-\mathbf{H}^{\mathrm{T}} \cdot\left[\mathbf{J}^{\mathrm{T}}-\mathbf{S}^{\mathrm{T}}\right] \cdot \boldsymbol{\lambda} \\
& =-\mathbf{H} \cdot \mathbf{g}_{\mathrm{R}} \cdot \frac{1}{2}\left[\mathbf{R}_{\mathrm{R}}+\mathbf{R}_{\mathrm{R}}^{\mathrm{T}}\right] \cdot \boldsymbol{f}_{\mathrm{R}}+\mathbf{H} \cdot\left[\mathbf{J}+\mathbf{g}_{\mathrm{R}} \cdot \mathbf{R}_{\mathrm{R}}^{\mathrm{T}} \cdot \mathbf{g}_{\mathrm{R}}^{\mathrm{T}}\right] \cdot \boldsymbol{\lambda}
\end{aligned}
$$




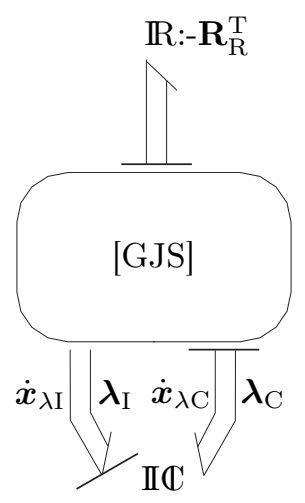

Figure 5: Bond graph translation of the term $\boldsymbol{\Lambda}_{\lambda}$ contribution in equation (20)

where $\frac{\partial}{\partial \boldsymbol{x}}$ now denotes the partial derivative of a vector with respect to the $\boldsymbol{x}$ which is a matrix, composed in rows by the gradients of the vector components with respect to $\boldsymbol{x}$.

Before introducing the bond graph translation of this equation, the variable mapping $\boldsymbol{x}_{\lambda}=\mathbf{H}^{-1} \cdot \boldsymbol{\lambda}$ is carried out ( $\mathbf{H}$ is definite positive by hypothesis and thus invertible). This gives:

$$
\begin{aligned}
\dot{\boldsymbol{x}}_{\lambda} & =-\mathbf{g}_{\mathrm{R}} \cdot \frac{1}{2}\left[\mathbf{R}_{\mathrm{R}}+\mathbf{R}_{\mathrm{R}}^{\mathrm{T}}\right] \cdot \boldsymbol{f}_{\mathrm{R}}+\left[\mathbf{J}+\mathbf{g}_{\mathrm{R}} \cdot \mathbf{R}_{\mathrm{R}}^{\mathrm{T}} \cdot \mathbf{g}_{\mathrm{R}}^{\mathrm{T}}\right] \cdot \mathbf{H} \cdot \boldsymbol{x}_{\lambda} \\
& =\boldsymbol{\Lambda}_{x}+\boldsymbol{\Lambda}_{\lambda}
\end{aligned}
$$

with $\boldsymbol{\Lambda}_{x}=-\mathbf{g}_{\mathrm{R}} \cdot \frac{1}{2}\left[\mathbf{R}_{\mathrm{R}}+\mathbf{R}_{\mathrm{R}}^{\mathrm{T}}\right] \cdot \boldsymbol{f}_{\mathrm{R}}$ and $\boldsymbol{\Lambda}_{\lambda}=\left[\mathbf{J}+\mathbf{g}_{\mathrm{R}} \cdot \mathbf{R}_{\mathrm{R}}^{\mathrm{T}} \cdot \mathbf{g}_{\mathrm{R}}^{\mathrm{T}}\right] \cdot \mathbf{H} \cdot \boldsymbol{x}_{\lambda}$.

The reason for this variable mapping is that the co-state vector $\boldsymbol{\lambda}$ is analog to a co-energy vector in bond graph language while the vector $\boldsymbol{x}_{\lambda}$ is analog to an energy vector. Since this new vector is not the co-state vector and to distinguish it from the state vector $\boldsymbol{x}$, it is called optimizing-state vector.

A first inspection shows clearly that the second term $\boldsymbol{\Lambda}_{\lambda}$ of the equation (20) second member corresponds to the eigen dynamics of the optimizing-state while the first term $\boldsymbol{\Lambda}_{x}$, is related to the coupling between both the state and optimizing-state systems. Concerning the term $\boldsymbol{\Lambda}_{\lambda}$, it is not difficult to see that it is closely analog to the expression of the state equations as equation (21) shows.

$$
\boldsymbol{x}:\left[\mathbf{J}-\mathbf{g}_{\mathrm{R}} \cdot \mathbf{R}_{\mathrm{R}} \cdot \mathbf{g}_{\mathrm{R}}^{\mathrm{T}}\right] \cdot \mathbf{H} \longrightarrow \boldsymbol{x}_{\lambda}:\left[\mathbf{J}-\mathbf{g}_{\mathrm{R}} \cdot\left(-\mathbf{R}_{\mathrm{R}}^{\mathrm{T}}\right) \cdot \mathbf{g}_{\mathrm{R}}^{\mathrm{T}}\right] \cdot \mathbf{H}
$$

In consequence the Fig. 4 bond graph structure embedding the multiport storage element, the multiport R-element, and the junction structure between these two can be reproduced to represent the term $\boldsymbol{\Lambda}_{\lambda}$ contributing to $\dot{\boldsymbol{x}}_{\lambda}$ (Fig. 5) just by changing $\mathbf{R}_{\mathrm{R}}$ into $-\mathbf{R}_{\mathrm{R}}^{\mathrm{T}}$.

An inspection of the term $\boldsymbol{\Lambda}_{x}$ in equation (20) shows that its contribution stems from the previously introduced multiport R-element through the junction structure transformation characterized by $\mathbf{g}_{\mathrm{R}}$. So the term $\frac{1}{2}\left[\mathbf{R}_{\mathrm{R}}+\mathbf{R}_{\mathrm{R}}^{\mathrm{T}}\right] \cdot \boldsymbol{f}_{\mathrm{R}}$ is calculated from the vector $\boldsymbol{f}_{\mathrm{R}}$ and contributes to the effort vector on the same R-element multibond. This results in the Fig. 6 bond graph translation that is a concatenation of the Fig. 4 and 5 bond graphs where the multiport R-elements have been replaced by a global multiport R-element. This R-element is characterized by the two matrices $\mathbf{R}_{R}$ and $-\mathbf{R}_{\mathrm{R}}^{\mathrm{T}}$ arranged in block diagonal, and by the complementary lower extra diagonal submatrix $\frac{1}{2}\left[\mathbf{R}_{\mathrm{R}}+\mathbf{R}_{\mathrm{R}}^{\mathrm{T}}\right]$ (the minus sign in the term $\boldsymbol{\Lambda}_{x}$ in equation 20 stems from the junction structure, in the same way as the minus sign in front of the matrix $\mathbf{S}$ in equation 16). This multiport R-element represents the coupling between the state and optimizing-state equations. It is proposed to call the added bond graph representation optimizing-bond graph, mirroring to some extent the original one.

It remains now to treat equation (18) which corresponds to the Euler equation with respect to the control vector $\boldsymbol{u}$.

First it is re-written as follows:

$$
\mathbf{R}_{u}^{-1} \cdot \boldsymbol{u}+\mathbf{g}^{\mathrm{T}} \cdot \boldsymbol{\lambda}=\mathbf{0}
$$




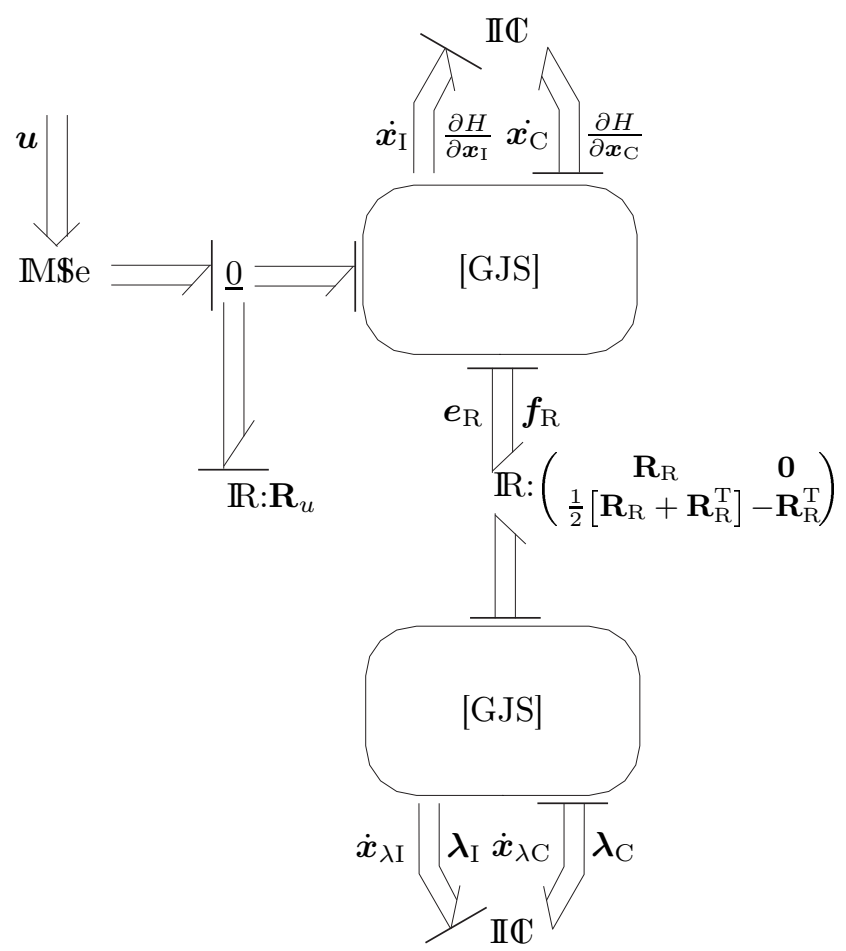

Figure 6: Bond graph translation of both state and optimizing-state equations (16) and (20)

This equation can be interpreted as a flow vector balance between a vector stemming from the control vector $\boldsymbol{u}$ in the original system and a vector coming from the vector $\boldsymbol{\lambda}$ through the junction structure transformation characterized by $\mathbf{g}$. This balance is translated by mirroring, in the optimizing bond graph, the left-hand side part of the Fig. 4 model bond graph between the junction structure and the modulated effort source (figure 7). Likewise, the multiport R-elements are concatenated into a global multiport R-element characterized by the matrix (23).

$$
\left(\begin{array}{cc}
\mathbf{R}_{u} & \mathbf{0} \\
\mathbf{R}_{u} & -\mathbf{R}_{u}
\end{array}\right)
$$

Now by imposing simultaneously the balance of the two flow vectors and a null effort vector to the optimizing bond graph 0-junction array (figure 7), the Euler equation (18) with respect to $\boldsymbol{u}$ is verified as the following development proves it using the figure 7 vector notations:

$$
\begin{array}{ll}
\boldsymbol{e}_{\mathrm{opt}}=\mathbf{R}_{u} \cdot \boldsymbol{f}-\mathbf{R}_{u} \cdot \boldsymbol{f}_{\mathrm{opt}}=\boldsymbol{f}-\boldsymbol{f}_{\mathrm{opt}} & \text { from the second vector characteristic law of the R-element } \\
\boldsymbol{f}_{\mathrm{opt}}=-\mathbf{g}^{\mathrm{T}} \cdot \boldsymbol{\lambda} & \text { from the flow vector balance } \\
\boldsymbol{f}=\mathbf{R}_{u}^{-1} \cdot \boldsymbol{e}=\mathbf{R}_{u}^{-1} \cdot \boldsymbol{u} & \text { from the first vector characteristic law of the R-element } \\
\text { so } & \\
\boldsymbol{e}_{\mathrm{opt}}=\mathbf{R}_{u}^{-1} \cdot \boldsymbol{u}+\mathbf{g}^{\mathrm{T}} \cdot \boldsymbol{\lambda}=\mathbf{0} &
\end{array}
$$

It is justified here not to have calculated a priori the optimal controls from the Euler equations in terms of the vector $\boldsymbol{u}$ components.

Finally the bond graph element that enables both a null effort vector and a balance of the two flow vectors to be imposed onto a 0-junction array is a multiport double source null effort vector and null flow vector. It is connected to the bottom 0 -junction array of the figure 7 bond graph. Such an element initializes bicausality $[24,25]$ propagation in the bond graph and thus requires the presence likewise of a multiport double detector $[11,12,13]$. In the mathematical formulation of the optimal control design problem, the role of the control 


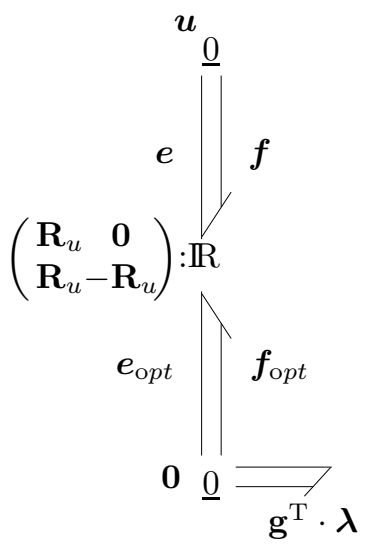

Figure 7: Bond graph translation of Euler equation (18) with respect to $\boldsymbol{u}$

vector $\boldsymbol{u}$ is changed into an output vector while the power conjugate vector $\boldsymbol{y}$ keeps its original output role. Thus the multiport double detector replaces the original multiport MSe element in the figure 4 bond graph.

The final generic causal bond graph representation, in the partial dualization context, of the given optimal control problem, is displayed in figure 8 and proves, by applying reverse dualization (see appendix A), the proposition and so the effectiveness of the section 2 procedure.

\section{End of the proof}

At this point it is worthwhile noting that, in the application of the procedure, there is no need to transform the original bond graph model into a partially dualized version. Dualization has been used in this section only for a concise demonstration reason and the procedure applies with a traditional bond graph representation displaying every element of the set $\{\mathrm{Se}, \mathrm{Sf}, \mathrm{I}, \mathrm{C}, \mathrm{R}, \mathrm{TF}, \mathrm{GY}, 1,0\}$. The correspondence between the different multiport R-element matrices of the figure 8 generic bond graph and that of figure 2-b is developed in appendix A. Also only certain of the inputs and certain of the dissipation phenomena can be involved in the optimal control problem and more precisely in the performance index. It is not difficult to see that the procedure still applies. In this case, the corresponding multiport R-elements are left uncoupled in the step 3 procedure for the uninvolved dissipation phenomena. Likewise, for the inputs excluded from the optimal control design problem, the multiport source elements are not replaced by double detectors and no R-element is added at the procedure step 1 . The DC motor example presented in the next section illustrates such a case where not all the dissipation phenomena are taken into consideration in the integral performance index.

\section{Illustrative example: a DC motor}

A DC motor model is presented in Fig. 9. It consists of the armature circuit composed of a voltage source $u$, a resistance $\mathrm{R}$ and an inductance $\mathrm{L}$. The electromechanical coupling is characterized by the torque constant $\mathrm{k}_{\mathrm{c}}$ and on the mechanical side, the rotor inertia $\mathrm{J}_{\mathrm{m}}$, viscous friction on rotor (parameter $\mathrm{b}_{\mathrm{m}}$ ), a reduction gear (parameter $1 / \mathrm{N}$ ) with a stiffness $\mathrm{k}$, the load inertia $\mathrm{J}_{\mathrm{c}}$ and a viscous friction on load shaft (parameter $\mathrm{b}_{\mathrm{c}}$ ) are taken into consideration. The model is linear and, in the optimal control context, the aim is, with given initial conditions at $t_{0}$ and given final conditions at $t_{f}$, to determinate $u$ with the integral performance index (24) that corresponds to the input and a certain dissipative energy minimization.

$$
V=\int_{\mathrm{t}_{0}}^{\mathrm{t}_{\mathrm{f}}} \frac{1}{2}\left(\frac{u^{2}}{\mathrm{R}_{u}}+P_{\mathrm{R}}+P_{\mathrm{b}_{\mathrm{c}}}\right) d t
$$

where $\mathrm{R}_{u}$ is a control weighted factor, $P_{\mathrm{R}}$ is the electrical power loss and $P_{\mathrm{b}_{\mathrm{c}}}$ the power dissipation on the load shaft.

The bond graph representation of this DC motor model is given in Fig. 10. It shows the MSe element for the voltage source, three I-elements for the three energy storage phenomena, associated respectively to 


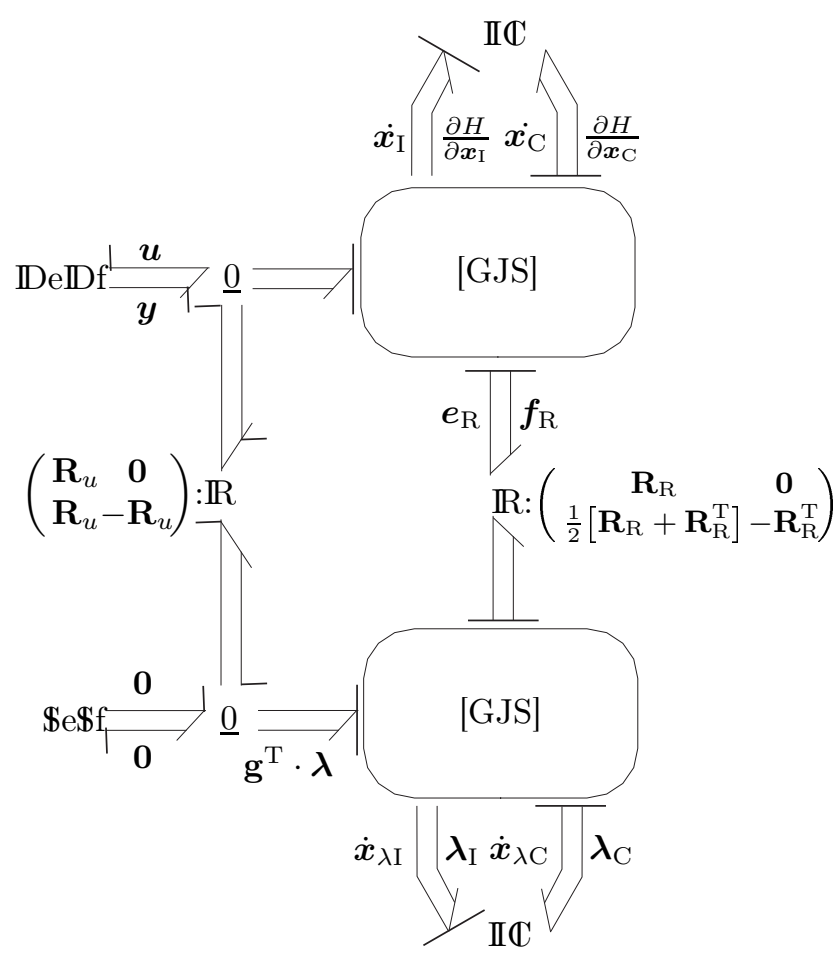

Figure 8: Generic bond graph representation of an optimal control problem

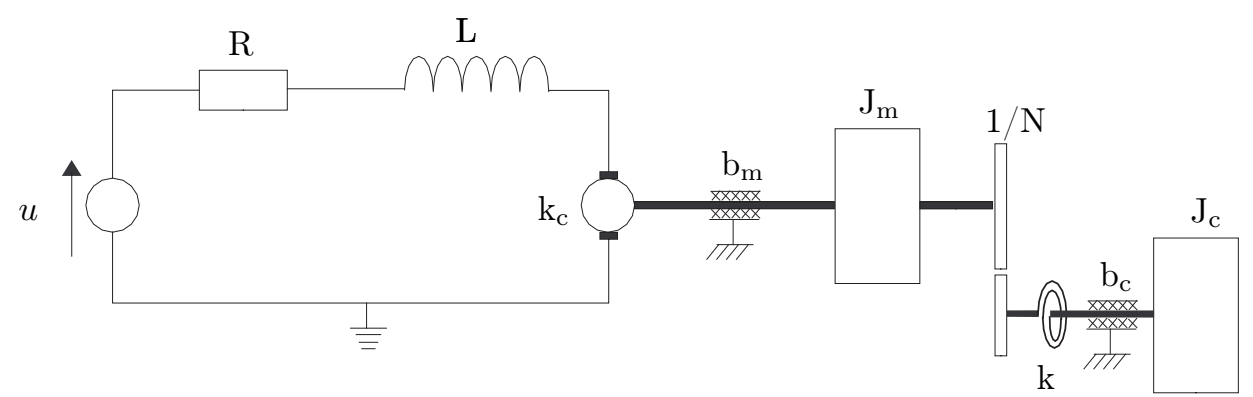

Figure 9: A DC motor model 


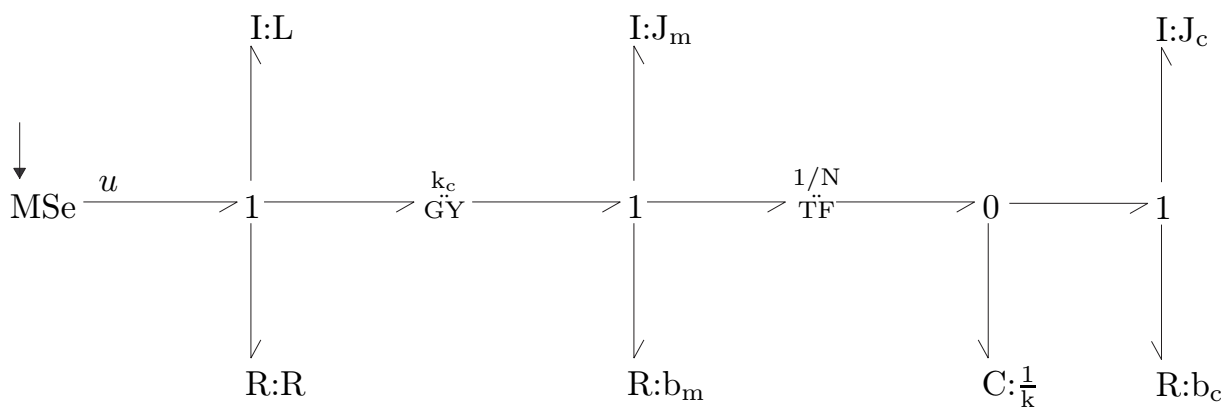

Figure 10: DC motor bond graph representation

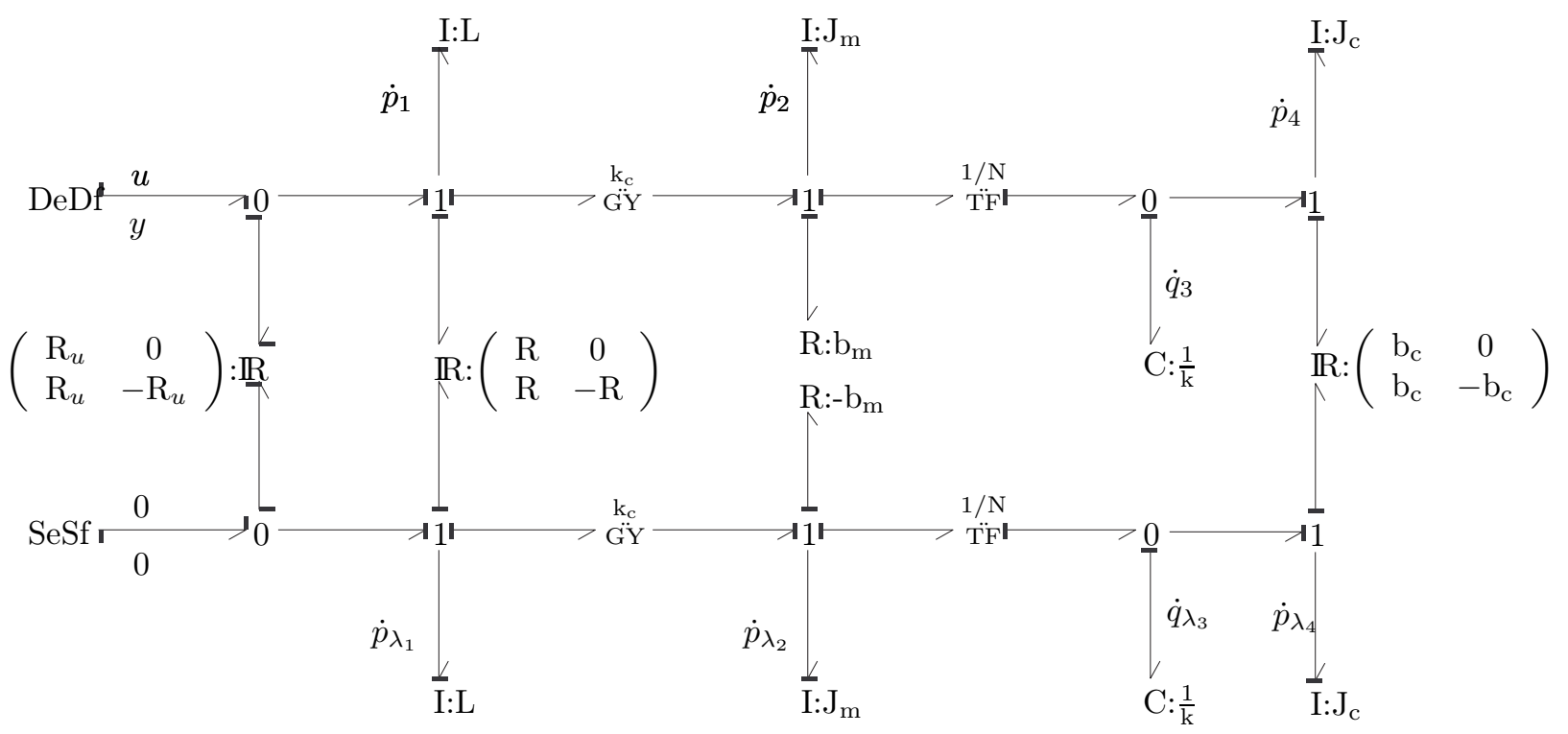

Figure 11: Bicausal bond graph representation of the DC motor optimal control problem

the magnetic energy, and the kinetic energies of the rotor and the load shaft motions, a C-element for the energy storage associated to the reduction gear stiffness, and three R-elements for the dissipation phenomena in the electrical circuit, on the rotor and the load shafts. The GY-element represents the electromechanical coupling and the TF-element is associated to the power conserving coupling in the reduction gear.

The equation (24) performance index involves the left-hand and right-hand side R-elements only.

The section 2 procedure application and, more particularly, the first five steps provide the Fig. 11 bicausal bond graph representation.

The bicausality assignment, as displayed on the Fig. 11 bond graph, enables the optimal control system 
(25) to be obtained. This constitutes the final step of the section 2 procedure.

$$
\left\{\begin{array}{l}
\dot{p}_{1}=-\frac{\mathrm{R}}{\mathrm{L}} p_{1}-\frac{\mathrm{k}_{\mathrm{c}}}{\mathrm{J}_{\mathrm{m}}} p_{2}-\frac{\mathrm{R}_{u}}{\mathrm{~L}} p_{\lambda_{1}} \\
\dot{p}_{2}=\frac{\mathrm{k}}{\mathrm{L}} p_{1}-\frac{\mathrm{b}}{\mathrm{J}_{\mathrm{m}}} p_{2}-\frac{\mathrm{k}}{\mathrm{N}} q_{3} \\
\dot{q}_{3}=\frac{1}{\mathrm{~J}_{\mathrm{m}} \mathrm{N}} p_{2}-\frac{1}{\mathrm{~J}_{\mathrm{c}}} p_{4} \\
\dot{p}_{4}=\mathrm{k} q_{3}-\frac{\mathrm{b}_{\mathrm{c}}}{\frac{\mathrm{J}}{\mathrm{J}_{\mathrm{c}}}} p_{4} \\
\dot{p}_{\lambda_{1}}=-\frac{\mathrm{R}}{\mathrm{L}} p_{1}+\frac{\mathrm{R}}{\mathrm{L}} p_{\lambda_{1}}-\frac{\mathrm{k}_{\mathrm{c}}}{\mathrm{J}_{\mathrm{m}}} p_{\lambda_{2}} \\
\dot{p}_{\lambda_{2}}=\frac{\mathrm{k} \mathrm{c}}{\mathrm{L}} p_{\lambda_{1}}+\frac{\mathrm{b} \mathrm{m}}{\mathrm{J}_{\mathrm{m}}} p_{\lambda_{2}}-\frac{\mathrm{k}}{\mathrm{N}} q_{\lambda_{3}} \\
\dot{q}_{\lambda_{3}}=\frac{1}{\mathrm{~J}_{\mathrm{m}} \mathrm{N}} p_{\lambda_{2}}-\frac{1}{\mathrm{~J}_{\mathrm{c}}} p_{\lambda_{4}} \\
\dot{p}_{\lambda_{4}}=-\frac{\mathrm{b}_{\mathrm{c}}}{\mathrm{J}_{\mathrm{c}}} p_{4}+\mathrm{k} q_{\lambda_{3}}+\frac{\mathrm{b}_{\mathrm{c}}}{\mathrm{J}_{\mathrm{c}}} p_{\lambda_{4}} \\
u=-\frac{\mathrm{R} u}{\mathrm{~L}} p_{\lambda_{1}} \\
y=\frac{1}{\mathrm{~L}} p_{1}-\frac{1}{\mathrm{~L}} p_{\lambda_{1}}
\end{array}\right.
$$

The application of the Pontryagin Maximum Principle leads to the same result with analytical developments compared to the bond graph graphical approach for deriving the equations.

The next section shows a number of key features of the approach of this paper for an optimal control bond graph formulation.

\section{$5 \quad$ Key features}

Sufficient condition The optimal system (13), stemming from the Euler-Lagrange conditions on the Pontryagin function (12), represents necessary conditions for an optimum existence. To determine whether it is a minimum, a maximum or none of them, one has to inspect the second variation of the functional in the performance index. This can be achieved by studying the definiteness of the matrix $\Pi$ (equation 26) [4, 26].

$$
\boldsymbol{\Pi}=\left(\begin{array}{ll}
\frac{\partial^{2} H_{\mathrm{p}}}{\partial \boldsymbol{x}^{2}} & \frac{\partial^{2} H_{\mathrm{p}}}{\partial \boldsymbol{x} \partial \boldsymbol{u}} \\
\frac{\partial^{2} H_{\mathrm{p}}}{\partial \boldsymbol{x} \partial \boldsymbol{u}} & \frac{\partial^{2} H_{\mathrm{p}}}{\partial \boldsymbol{u}^{2}}
\end{array}\right)_{\boldsymbol{u}=\boldsymbol{u}_{\mathrm{opt}}}
$$

where $H_{\mathrm{p}}$ is the Pontryagin function, $\boldsymbol{x}$ the state vector, $\boldsymbol{u}$ the control vector, and subscript ' $\boldsymbol{u}=\boldsymbol{u}_{\mathrm{opt}}$ ' indicating that the terms are evaluated for the optimal control.

For the optimal control problem considered in this paper, the Pontryagin function (15) is expanded using (7) to give:

$$
H_{\mathrm{p}}=\frac{1}{2} \boldsymbol{u}^{\mathrm{T}} \cdot \mathbf{R}_{u}^{-1} \cdot \boldsymbol{u}+\frac{1}{2} \boldsymbol{x}^{\mathrm{T}} \cdot \mathbf{H} \cdot \mathbf{g}_{\mathrm{R}} \cdot \mathbf{R}_{\mathrm{R}} \cdot \mathbf{g}_{\mathrm{R}}^{\mathrm{T}} \cdot \mathbf{H} \cdot \boldsymbol{x}+\boldsymbol{\lambda}^{\mathrm{T}} \cdot[[\mathbf{J}-\mathbf{S}] \cdot \mathbf{H} \cdot \boldsymbol{x}+\mathbf{g} \cdot \boldsymbol{u}]
$$

The expression of matrix $\Pi$ is in this case:

$$
\boldsymbol{\Pi}=\left(\begin{array}{cc}
\mathbf{Q} & \mathbf{0} \\
\mathbf{0} & \mathbf{R}_{u}
\end{array}\right)_{\boldsymbol{u}=\boldsymbol{u}_{\mathrm{opt}}}
$$

where $\mathbf{Q}=\mathbf{H} \cdot \mathbf{g}_{\mathrm{R}} \cdot \mathbf{R}_{\mathrm{R}} \cdot \mathbf{g}_{\mathrm{R}}^{\mathrm{T}} \cdot \mathbf{H}=\mathbf{H S H}$ using equation (9).

By hypothesis, $\mathbf{R}_{u}$ is diagonal with positive elements. Thus it is sufficient to study the matrix $\mathbf{Q}[27,28]$. In the particular case of the multiport R-elements with positive definite characteristic matrices, a minimum can be concluded.

Instability behavior Instability is a known feature of the optimal control system (13) [29]. Without getting in depth in a theoretical demonstration, this feature can be easily viewed by inspecting the corresponding state matrix (Equ. 16 and 17). In fact the trace $\mathrm{tr}_{\mathrm{opt}}$ of this matrix is given by :

$$
\begin{aligned}
\operatorname{tr}_{\mathrm{opt}} & =\operatorname{tr}([\mathbf{J}-\mathbf{S}] \cdot \mathbf{H})-\operatorname{tr}\left(\mathbf{H} \cdot\left[\mathbf{J}^{\mathrm{T}}-\mathbf{S}^{\mathrm{T}}\right]\right) \\
& =\operatorname{tr}([\mathbf{J}-\mathbf{S}] \cdot \mathbf{H})-\operatorname{tr}([\mathbf{J}-\mathbf{S}] \cdot \mathbf{H}) \\
& =0,
\end{aligned}
$$


since for any matrix $\mathbf{M}, \operatorname{tr}(\mathbf{M})=\operatorname{tr}\left(\mathbf{M}^{\mathrm{T}}\right)$.

Now the trace of a matrix is invariant and so is also the sum of the eigenvalues. For the optimal control system (16) and (17) this means that if at least one eigenvalue with a negative real part exists, then one with an opposite real part as well exists. It can be concluded that this system is unstable.

This feature is very important for numerical simulation considerations when an indirect approach is followed in the optimal control problem resolution. It is worthwhile noting also that the boundary conditions have no incidence on this unstability result.

Ordinary form of the differential equations The state space system Equ. (8), considered for the proof of the procedure effectiveness, has been implicitly supposed to be an Ordinary Differential Equation (ODE) system. This corresponds to a causal bond graph with no zero-order causal path (ZCP) that would otherwise add algebraic equations to the model (index of the Differential Algebraic Equation (DAE) system greater than 0) [30]. In fact the procedure still applies to a large extent when zero-order causal paths appear in the causal bond graph. The condition is that these ZCPs must be solvable with respect to an algebraic variable so that the DAE system can be transformed into an ODE one. In fact this is the great majority of cases. One unfavourable case, given in [30], is when ZCPs of class 3 or 4 touch ZCPs of class 1. This corresponds to causal loops between one storage element in integral causality and one in derivative causality, touching a causal mesh or a causal cycle of loop gains different from 1 . This rare case is thus implicitly excluded from the proposed procedure of this paper.

\section{Conclusion}

In this paper a procedure and the proof of its effectiveness has been given for introducing an optimal control formulation into the bond graph language. The class of the optimal control problems presented concerns linear time invariant MIMO systems where the integral performance index corresponds to inputs and a dissipative energy minimisation. The procedure enables the formulation to be set up exclusively at a graphical (namely bond graph) level. The proof of its effectiveness uses the Pontryagin principle applied to the port-Hamiltonian formulation of the system.

The main contribution of this paper is to provide an alternative way to derive an optimal control system for the type of optimisation problems specified. However no method has been given for solving the system of equations obtained. The corresponding approach is indirect and further investigations are required to exploit the bond graph stemming from the procedure at a numerical simulation level. Thus the key issue is now to couple numerical methods designed for two point boundary-value problems. The shooting or the multiple shooting method, the collocation method or the finite differences method are examples of numerical techniques for the two point boundary-value problem [26].

As already stated in the introduction, a bond graph formulation of an optimisation problem brings a new view and a graphical visualization of this type of problem. This can facilitate the analysis of the coupling between the state space and the co-state one, and also facilitate the interpretation of the state and co-state variable roles or the output role assigned to the inputs to be determined. In particular the bond graph representation shows clearly that the co-state variables can be interpreted as co-energy variables and that, by appropriate mapping, energy-like variables corresponding to them can be made explicit on bond graph storage elements. Also the bond graph formulation shows clearly the appearence of the adjoint system [31,32] that has already been pointed out in previous works [33,34]. This confers some generic features of certain parts of the proposed procedure with regard to other types of optimisation problems.

A set of perspectives opens the extension of the procedure to time variant and then to non linear problems, to other boundary conditions and to performance indexes other than those based on energy dissipation. Practical problems always involve inequality constraints. They correspond to the fact that certain variables cannot be physically greater than given values. This can be considered for state variables and for inputs. Works has to be done to introduce these constraints into the bond graph representation.

Finally, though the work in this paper has been presented in the context of an optimal control problem formulation in bond graph language, it must be viewed rather as the problem of the input determination corresponding to a dynamic optimization problem. It is completely equivalent from the mathematical point 
of view but the authors' objective in the future is to couple this procedure to a sizing methodology based on an inverse model approach. In this design context the aim is to specify the technological components that constitute an actuacting line $[11,12,13]$.

\section{References}

[1] Marquis-Favre, W., Chereji, B., Thomasset, D., Scavarda, S. Bond graph representation of an optimal control problem: the dc motor example. In ICBGM'05 International Conference of Bond Graph Modelling and simulation, pages 239-244, New Orleans, USA, January 23-27 2005.

[2] Naslin, P. Introduction à la commande optimale. Bibliothèque de l'automaticien. Dunod, Paris, 1966.

[3] Pun, L. Introduction to optimization Practice. John Wiley \& Sons, Inc., New York, 1969.

[4] Naidu, D. S. Optimal control systems. CRC Press, 2003.

[5] Cabanellas, J. M., Félez, J., Vera, C. A formulation of the sensitivity analysis for dynamic systems optimization based on pseudo bond graphs. In ICBGM'95 International Conference of Bond Graph Modelling and simulation, pages 135-144, Las Vegas, UsA, January 15-18 1995.

[6] Cabanellas, J. M., Félez, J. Dynamic systems optimization based on pseudo bond graph. In ICBGM'99 International Conference on Bond Graph Modeling and Simulation, pages 50-55, San Francisco, UsA, January 17-20 1999.

[7] Roe, P. H., Thoma, J. U. A new bond graph approach to sensitivity analysis. In Third IMACS Symposium on Mathematical Modelling, pages 743-746, Vienne, Austria, February 2000.

[8] Gawthrop, P. J. Sentivity bond graphs. Journal of The Franklin Institute, 337:907-922, 2000.

[9] Gawthrop, P. J., Ronco, E. Estimation and control of mechatronic systems using sensitivity bond graphs. Control Engineering Practice, (8):1237-1248, 2000.

[10] Fotsu-Ngwompo, R. Contribution au Dimensionnement des Systèmes sur des Critères Dynamiques et Energétiques - Approche par Bond Graph. Ph. d. thesis, Institut National des Sciences Appliquées de Lyon, 1997.

[11] Fotsu-Ngwompo, R., Scavarda, S. Dimensioning problems in system design using bicausal bond graphs. Simulation Practice and Theory, 7:577-587, 1999.

[12] Fotsu-Ngwompo, R., S. Scavarda, Thomasset, D. Physical model-based inversion in control systems design using bond graph representation. part 1: Theory. Proceedings of the IMECHE Part I Journal of Systems and Control Engineering, 215:95-103, 2001.

[13] Fotsu-Ngwompo, R., S. Scavarda, Thomasset, D. Physical model-based inversion in control systems design using bond graph representation. part 2: Applications. Proceedings of the IMECHE Part I Journal of Systems and Control Engineering, 215:105-112, 2001.

[14] Maschke, B. M., van der Schaft, A. J., Breedveld, P. C. An intrinsic hamiltonian formulation of network dynamics: Non-standard poisson structures and gyrators. Journal of The Franklin Institute, 329:923966, 1992 .

[15] Maschke, B., van der Schaft, A. J. Port-controlled hamiltonian systems: Modelling origins and systemtheoretic properties. In $2^{\text {nd }}$ IFAC NOLCOS, pages 282-288, Bordeaux, 1992.

[16] van der Schaft, A. J., Maschke, B. Hamiltonian formulation of bond graphs. In Ecole d'été d'Automatique de Grenoble, Session 22: Modélisation Géométrique et Commande des Systèmes Physiques, Grenoble, France, 17-21 septembre 2001. Brogliato, B. 33 pages. 
[17] van der Schaft, A. J., Maschke, B. Port-hamiltonian systems: towards a theory for geometric modeling and control of complex physical systems. In Ecole d'été d'Automatique de Grenoble, Session 22 : Modélisation Géométrique et Commande des Systèmes Physiques, Grenoble, France, 17-21 septembre 2001. Brogliato, B. 20 pages.

[18] Breedveld, P. C. A definition of the multibond graph language. In S. Tzafestas and P. Borne, editors, ImACs Transactions on Scientific Computing, Complex and Distributed Systems: Analysis, Simulation and Control, volume 4, pages 69-72, Amsterdam, 1986. North-Holland Publ. Comp.

[19] Crandall, S. H., Karnopp, D. C., Kurtz, E. F., Pridmore-Brown, D. C. Dynamics of mechanical and electro-mechanical systems. McGraw-Hill, New-York, 1968.

[20] Karnopp, D.C., Margolis, D.L., Rosenberg, R.C. System Dynamics: Modeling and Simulation of Mechatronic Systems. John Wiley \& Sons, New York, 2000.

[21] Breedveld, P. C. Multibond graph elements in physical systems theory. Journal of The Franklin Institute, $319(1 / 2): 1-36,1985$.

[22] Schultz, D. G. and Melsa, J. State Functions and Linear Control Systems. McGraw Hill Book Company, 1967.

[23] Takahashi, Y., Rabins M., Auslander, D. Control and Dynamic Systems. Addison-Wesley ed., 1972.

[24] Gawthrop, P. J. Bicausal bond graphs. In ICBGM'95 International Conference on Bond Graph Modeling and Simulation, pages 83-88, Las Vegas, USA, January 15-18 1995.

[25] Gawthrop, P. J. Physical interpretation of inverse dynamics using bicausal bond graphs. Journal of The Franklin Institute, 337:743-769, 2000.

[26] Agrawal, S. K. and Fabien, B. C. Optimization of dynamic systems, volume 70 of Solid Mechanics and its Applications. Kluwer Academic Publishers, Dordrecht, Boston, London, 1999.

[27] Gantmacher, F. R. Théorie des matrices : tome 1, théorie générale. Collection universitaires de mathématiques. Paris, dunod edition, 1966.

[28] Ayres, F. Matrices : cours et problèmes. Série Schaum. Paris, ediscience s. a. edition, 1974.

[29] Lewis, F. L. and Syrmos, V. L. Optimal control. John Wiley \& Sons, Inc., New York, second edition, 1995.

[30] van Dijk, J. On the role of bond graph causality in modelling mechatronic systems. Ph.d. thesis: Electrical engineering, University of Twente, Enscheden, Netherlands, 1994.

[31] Zadeh, L. A., and Desoer, C. A. Linear system theory. The state space approach. McGraw-Hill Book Company, New York, 1963.

[32] Kailath, T. Linear Systems. Prentice Hall, Inc., Englewood Cliffs, NJ, 1980.

[33] Xia, X. Contribution à l'Analyse du Comportement Dynamique et Energétique d'un Système Linéaire et Stationnaire Modélisé par un Bond Graph Bicausal. Ph. d. thesis, Institut National des Sciences Appliquées de Lyon, 2000.

[34] Xia, X., Scavarda, S. Adjoint system by using the representation of bond graph. In ICBGM '01 International Conference on Bond Graph Modeling and Simulation, pages 15-20, Phoenix, USA, January 07-11 2001. 
(a)

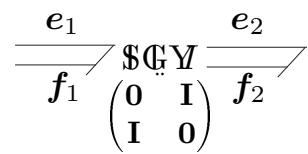

(b)

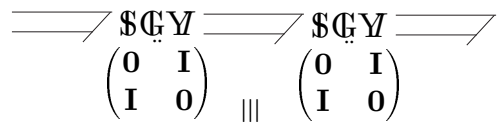

Figure 12: Symplectic gyrator: (a) representation, (b) one property

(a)

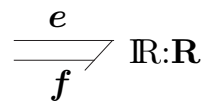

III

(b)

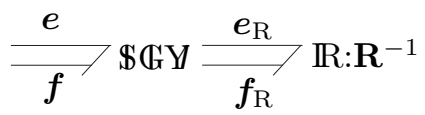

Figure 13: Dualization of the multiport R-elements: (a) original representation, (b) dualized representation

\section{Appendix}

\section{A Partial dualization}

The generic bond graph representation used for the proof of the proposed procedure is based on the dualization concept [21]. Dualization is realized by a symplectic gyrator or unit gyrator (Fig. 12-a) and enables the nature of the power variables to be exchanged. The implicit constitutive relation of this element is given by:

$$
\left(\begin{array}{l}
e_{1} \\
e_{2}
\end{array}\right)-\left(\begin{array}{ll}
\mathbf{0} & \mathbf{I} \\
\mathbf{I} & \mathbf{0}
\end{array}\right) \cdot\left(\begin{array}{l}
f_{1} \\
f_{2}
\end{array}\right)=\mathbf{0}
$$

where $\mathbf{I}$ is the identity matrix. One important property is that two symplectic multiport gyrators in series are equivalent to a multibond (Fig. 12-b). In the context of this paper, this multiport element has been used to simplify the development of the proof of the procedure effectiveness by means of dualization and partial dualization presented hereafter. Correspondence between the characteric matrices of original and dualized multiport R-elements are also detailed.

Dualization of the multiport R-elements Though not directly used in the proof, this dualization case enables this technique to be understood well. The symplectic multiport gyrator enables both the figures 13-a and 13-b partial bond graph representations to be used equivalently. The implicit constitutive relation equivalence is shown by:

$$
\begin{array}{ll} 
& \boldsymbol{e}-\mathbf{R} \cdot \boldsymbol{f}=\mathbf{0} \\
\Leftrightarrow & \boldsymbol{f}_{\mathrm{R}}-\mathbf{R} \cdot \boldsymbol{e}_{\mathrm{R}}=\mathbf{0} \\
\Leftrightarrow & \boldsymbol{e}_{\mathrm{R}}-\mathbf{R}^{-1} \cdot \boldsymbol{f}_{\mathrm{R}}=\mathbf{0}
\end{array}
$$




$$
\begin{aligned}
& \frac{e_{\mathrm{r}}}{f_{\mathrm{r}}} \\
& \text { (a) } \underset{\boldsymbol{e}_{\mathrm{c}}}{\boldsymbol{f}_{\mathrm{r}}} \mathbb{R}: \mathbf{R}=\left[\begin{array}{ll}
\mathbf{R}_{\mathrm{rr}} & \mathbf{R}_{\mathrm{rc}} \\
\mathbf{R}_{\mathrm{cr}} & \mathbf{R}_{\mathrm{cc}}
\end{array}\right] \\
& \text { III }
\end{aligned}
$$

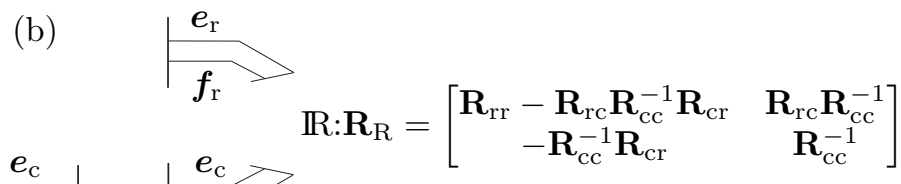

Figure 14: Partial dualization of the multiport R-elements: (a) original representation, (b) dualized representation

Partial dualization of the multiport R-elements Partial dualization is used here in a causal context. Concerning R-elements, it enables unique type of causality (here resistance causality) to be dealt with. Consider the figure 14-a causal multiport R-element collecting all individual 1-port or multiport R-elements of a general causal bond graph. Without loss of generality the ports of this multiport R-element have been classified in such a way that the first ones correspond to the resistance causality and the last ones to the conductance causality. The use of a multiport symplectic gyrator enables the conductance causality ports to be dualized (Fig. 14-b). The equivalence between both the R-element constitutive matrices is shown by:

$$
\begin{aligned}
&\left\{\begin{array}{l}
\boldsymbol{e}_{\mathrm{r}}-\mathbf{R}_{\mathrm{rr}} \cdot \boldsymbol{f}_{\mathrm{r}}-\mathbf{R}_{\mathrm{rc}} \cdot \boldsymbol{f}_{\mathrm{c}}=\mathbf{0} \\
\boldsymbol{e}_{\mathrm{c}}-\mathbf{R}_{\mathrm{cr}} \cdot \boldsymbol{f}_{\mathrm{r}}-\mathbf{R}_{\mathrm{cc}} \cdot \boldsymbol{f}_{\mathrm{c}}=\mathbf{0}
\end{array}\right. \\
& \Leftrightarrow \quad\left\{\begin{array}{l}
\boldsymbol{e}_{\mathrm{r}}=\left(\mathbf{R}_{\mathrm{rr}}-\mathbf{R}_{\mathrm{rc}} \mathbf{R}_{\mathrm{cc}}^{-1} \mathbf{R}_{\mathrm{cr}}\right) \cdot \boldsymbol{f}_{\mathrm{r}}+\mathbf{R}_{\mathrm{rc}} \mathbf{R}_{\mathrm{cc}}^{-1} \cdot \boldsymbol{e}_{\mathrm{c}} \\
\boldsymbol{f}_{\mathrm{c}}=-\mathbf{R}_{\mathrm{cc}}^{-1} \mathbf{R}_{\mathrm{cr}} \cdot \boldsymbol{f}_{\mathrm{r}}+\mathbf{R}_{\mathrm{cc}}^{-1} \cdot \boldsymbol{e}_{\mathrm{c}}
\end{array}\right. \\
& \Leftrightarrow \quad e_{\mathrm{R}}=\mathbf{R}_{\mathrm{R}} \cdot \boldsymbol{f}_{\mathrm{R}}
\end{aligned}
$$

with $\boldsymbol{e}_{\mathrm{R}}=\left(\begin{array}{l}\boldsymbol{e}_{\mathrm{r}} \\ \boldsymbol{f}_{\mathrm{c}}\end{array}\right)$ and $\boldsymbol{f}_{\mathrm{R}}=\left(\begin{array}{l}\boldsymbol{f}_{\mathrm{r}} \\ \boldsymbol{e}_{\mathrm{c}}\end{array}\right)$.

The following convention is recalled here: the power variable on the half arrow side is a flow while, on the other side, it is an effort.

Correspondence between $\mathbf{R}_{\mathrm{R}}^{\mathrm{T}}$ and $\mathbf{T}$ The proof of the procedure effectiveness shows the appearance of the matrix $\mathbf{R}_{\mathrm{R}}^{\mathrm{T}}$ in the optimizing bond graph (Fig. 8). It is essential to see the correspondence with the general bond graph representation (Fig. 2-b). Starting with the matrix $\mathbf{R}_{\mathrm{R}}^{\mathrm{T}}$ (Fig. 15-a) the corresponding matrix $\mathbf{T}$ is obtained by:

$$
\begin{aligned}
\left\{\begin{array}{l}
\boldsymbol{e}_{\mathrm{opt.r}}=\left(\mathbf{R}_{\mathrm{rr}}-\mathbf{R}_{\mathrm{rc}} \mathbf{R}_{\mathrm{cc}}^{-1} \mathbf{R}_{\mathrm{cr}}\right)^{\mathrm{T}} \cdot \boldsymbol{f}_{\mathrm{opt.r}}-\left(\mathbf{R}_{\mathrm{cc}}^{-1} \mathbf{R}_{\mathrm{cr}}\right)^{\mathrm{T}} \cdot \boldsymbol{e}_{\mathrm{opt} . \mathrm{c}} \\
\boldsymbol{f}_{\text {opt.c }}=\left(\mathbf{R}_{\mathrm{rc}} \mathbf{R}_{\mathrm{cc}}^{-1}\right)^{\mathrm{T}} \cdot \boldsymbol{f}_{\mathrm{opt.r}}+\left(\mathbf{R}_{\mathrm{cc}}^{-1}\right)^{\mathrm{T}} \cdot \boldsymbol{e}_{\mathrm{opt.c}}
\end{array}\right. \\
\Leftrightarrow\left\{\begin{array}{l}
\boldsymbol{e}_{\mathrm{opt.r}}=\mathbf{R}_{\mathrm{rr}}^{\mathrm{T}} \cdot \boldsymbol{f}_{\mathrm{opt.r}}-\mathbf{R}_{\mathrm{cr}}^{\mathrm{T}} \cdot \boldsymbol{f}_{\text {opt.c }} \\
\boldsymbol{e}_{\text {opt.c }}=-\mathbf{R}_{\mathrm{rc}}^{\mathrm{T}} \cdot \boldsymbol{f}_{\text {opt.r }}+\mathbf{R}_{\mathrm{cc}}^{\mathrm{T}} \cdot \boldsymbol{f}_{\text {opt.c }}
\end{array}\right.
\end{aligned}
$$

One important feature of the matrix $\mathbf{T}$ is that it is causality dependent. 


$$
\begin{aligned}
& \text { (a) } \underset{f_{\text {opt.r }}}{e_{\text {opt.r }}} \mathbb{R}: \mathbf{R}_{\mathrm{R}}^{\mathrm{T}}=\left[\begin{array}{cc}
\left(\mathbf{R}_{\mathrm{rr}}-\mathbf{R}_{\mathrm{rc}} \mathbf{R}_{\mathrm{cc}}^{-1} \mathbf{R}_{\mathrm{cr}}\right)^{\mathrm{T}} & -\left(\mathbf{R}_{\mathrm{cc}}^{-1} \mathbf{R}_{\mathrm{cr}}\right)^{\mathrm{T}} \\
\left(\mathbf{R}_{\mathrm{rc}} \mathbf{R}_{\mathrm{cc}}^{-1}\right)^{\mathrm{T}} & \left(\mathbf{R}_{\mathrm{cc}}^{-1}\right)^{\mathrm{T}}
\end{array}\right] \\
& \text { III } \\
& \text { (b) } \\
& \frac{e_{\text {opt.r }}}{f_{\text {opt.r }}}
\end{aligned}
$$

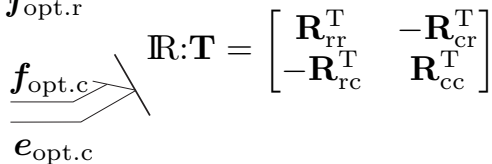

Figure 15: Bond graph correspondence between (a) $\mathbf{R}_{\mathrm{R}}^{\mathrm{T}}$ and (b) $\mathbf{T}$

Correspondence between $-\mathbf{R}_{\mathrm{R}}^{\mathrm{T}}$ and $-\mathbf{R}^{\mathrm{T}}$ Based on a development similar to that of the previous paragraph, it is not difficult to show that the matrix $-\mathbf{R}_{\mathrm{R}}^{\mathrm{T}}$ in the figure 8 bond graph corresponds to the matrix $-\mathbf{R}^{\mathrm{T}}$ in the figure 2-b bond graph representation.

In the particular case where the R-elements are 1-port elements, the matrix $\mathbf{R}$ is diagonal and $\mathbf{R}^{\mathrm{T}}=\mathbf{T}=$ R. This simplifies the final matrices that are thus causality independent.

Dualization of the multiport flow sources Dualization of the multiport flow sources is also used in the proof of the procedure effectiveness in order to simplify the causality cases. It is shown in figure 16 .

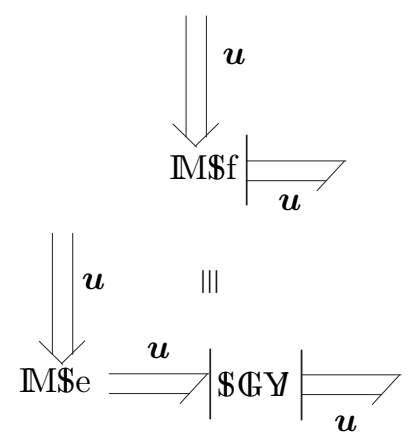

Figure 16: Dualization of the multiport flow sources

Dualization of the 0-junction arrays The correspondence between the figure 8 bond graph representation and the figure 2-b one requires the dualization of the 0-junction arrays as well. It is shown in figure 17 . This dualization is coordinated with the one of a multiport flow source and the one of a multiport R-element. 


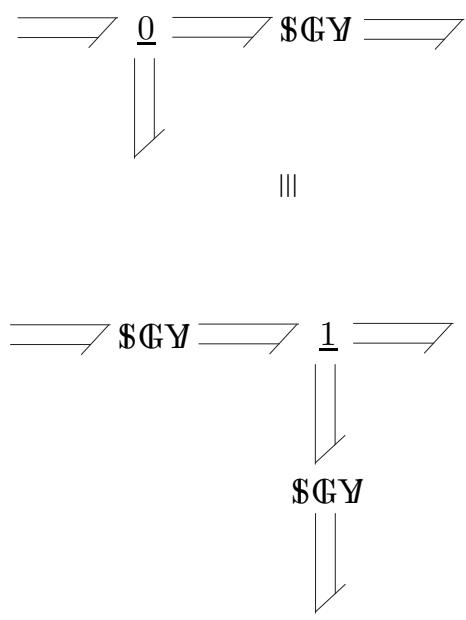

Figure 17: Dualization of the 0 -junction arrays

\section{B Bicausality}

The bicausality concept [24] is an extension of the causality concept corresponding to a strict mathematical point of view on a power bond. Considering the figure 18-a acausal bond graph representation of a power bond, this can be viewed as a graphical representation of a power connection between two subsystem power ports, thus constraining the power variables (effort and flow) to be identical. The mathematical representation of this power port connection can be expressed by the two implicit equations :

$$
\left\{\begin{array}{l}
e_{1}-e_{2}=0 \\
f_{1}-f_{2}=0
\end{array}\right.
$$

Causality corresponds to the organisation of these two equations with a strong physical interpretation and gives both the assignment possibilities of the figure 18-b causal power bonds. However inspection of the previous implicit equations shows that from a strict mathematical point of view, it is possible to have two other calculus schemes displayed in the figure 18-c called bicausal power bonds. This bicausal assignment has no physical interpretation. It only means that both power variables are mathematically determined at the same time by the same subsystem set of equations. The interest of using bicausality, and thus this assignment, becomes obvious for deriving inverse models [11, 25, 12, 13]. In the bicausity assignment the stroke is split into two half-strokes, one dedicated to effort assignment (half arrow opposite side), and the other one dedicated to flow assignment (half arrow side).

It remains now to introduce two new elements into the representation that, on one side, initiate a bicausality assignment, and, on the other side, properly terminate this bicausal assignment. The element from which bicausality starts is a double source (Fig. 19-a) and the element where bicausality terminates is a double sensor (Fig. 19-b).

Concerning the bond graph bicausal assignment, the element constraints are the same as for the causality assignment. The difference resides in the fact that effort and flow assignments are now uncoupled. In the bicausal bond graph, bicausality and causality coexist but junction constraints show that two bicausal paths cannot be adjacent. Finally bicausality is assigned from a double source to a double sensor, this means that these elements are necessarly present in pairs. 


$$
\begin{aligned}
& \text { (a) } \frac{e_{1} \quad e_{2}}{f_{1} \quad f_{2}} \\
& \text { (b) } \quad\left\{\begin{array} { l } 
{ e _ { 1 } : = e _ { 2 } } \\
{ f _ { 2 } : = f _ { 1 } }
\end{array} \quad \left\{\begin{array}{l}
e_{2}:=e_{1} \\
f_{1}:=f_{2}
\end{array}\right.\right. \\
& \longmapsto-1 \\
& \text { (c) } \quad\left\{\begin{array} { l } 
{ e _ { 1 } : = e _ { 2 } } \\
{ f _ { 1 } : = f _ { 2 } }
\end{array} \quad \left\{\begin{array}{l}
e_{2}:=e_{1} \\
f_{2}:=f_{1}
\end{array}\right.\right. \\
& \text { (c) }
\end{aligned}
$$

Figure 18: Power bond (a) acausal, (b) in causal assignment, and (c) in bicausal assignment

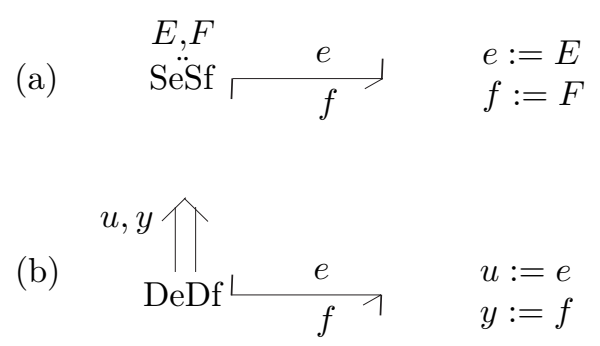

Figure 19: (a) Double source, (b) double sensor 